\title{
Applicability of Anisotropic Failure Criteria and Associated Application with Layered Rocks
}

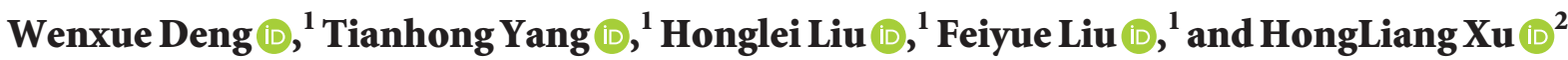 \\ ${ }^{1}$ Department of Mining Engineering, School of Resources and Civil Engineering, Northeastern University, \\ Shenyang 110819, China \\ ${ }^{2}$ Northern Engineering and Technology Corporation, Dalian 116600, China \\ Correspondence should be addressed to Honglei Liu; liuhonglei@mail.neu.edu.cn
}

Received 30 November 2020; Accepted 30 December 2020; Published 25 January 2021

Academic Editor: Zhijie Wen

Copyright (C) 2021 Wenxue Deng et al. This is an open access article distributed under the Creative Commons Attribution License, which permits unrestricted use, distribution, and reproduction in any medium, provided the original work is properly cited.

\begin{abstract}
This paper examines the applicability of four failure criteria applied to layered rocks, namely, Pariseau's model, linear empirical equation, nonlinear empirical equation (LEE and NLEE), and Jaeger's plane-of-weakness model (JPW). A database comprising 282 uniaxial compressive strength (UCS) tests and 1,273 triaxial tests conducted worldwide on layered rocks is compiled. Pariseau's model is classified as a continuous function, and LEE and NLEE, as well as JPW, are classified as piecewise functions. Nonlinear least squares and traversing methods are used to determine the global minima of the material coefficients in the two types of functions. The goodness of fit of these criteria for the database is evaluated. Two statistical parameters, namely, regression coefficient $\left(R^{2}\right)$ and mean absolute percentage error (MAPE) are used for precision assessment. The aforementioned methods and the criteria are further used for evaluating the surrounding rock stability of the eastern slope of Yanshan iron open-pit and the underground powerhouse of the Jinping I hydropower station. The results show that (1) Pariseau's model is not suitable for layered rocks when $n=2$, and the fit is worst in the condition of insufficient triaxial data when $n=1 ;(2)$ the MAPE of the empirical equation (LEE and NLEE) is within 20\%, with the best predictive accuracy under medium to low confining pressures, and the MPAE of JPW is within $40 \%$, with the best predictive accuracy under high confining pressures; and (3) calculation of the damage area of the slope and underground powerhouse and comparison with the actual damage on the site verify that the empirical equation has the best engineering prediction accuracy under medium to low confining pressure conditions.
\end{abstract}

\section{Introduction}

Sedimentary rocks characterized by layered structure account for two-thirds of the global land area. Several bedded sedimentary and foliated metamorphic rocks such as layered sandstone, siltstone, mudstone, shale, slate, schist and gneiss, and phyllite exhibit remarkable anisotropic strength behavior [1-4]. The compressive strength of layered rocks depends on the direction of loading because of the preferred fabric or the preferred discontinuity. The maximum strength is observed at $\beta=0^{\circ}$ or $\beta=90^{\circ}$, and the minimum is observed at $\beta=45-60^{\circ}$ ( $\beta$ is the angle between the horizontal direction and the anisotropic plane direction; Figure 1(a)). The strength response becomes nonlinear for a large range of confining pressures (Figure 1(b)).
Anisotropy can be found at different scales ranging from the intact specimens to rock mass. The excavation disturbance alters both the stress values and the principal stress direction, impacting the strength of anisotropic rocks $[6,7]$. The inherent or induced strength anisotropy of rocks should be completely accounted for predicting rock engineering performance. Therefore, classical isotropic failure criteria, which are only stress-dependent, cannot be used for layered rocks.

An ideal anisotropic failure criterion should not only predict the strength of the rock but also should be simple to use for rock engineering designers. The Mohr-Coulomb criterion is most widely used for isotropic rocks because its parameters are easily comprehended by engineers, whereas the Jaeger's plane of weakness model (JPW) [8] is most 


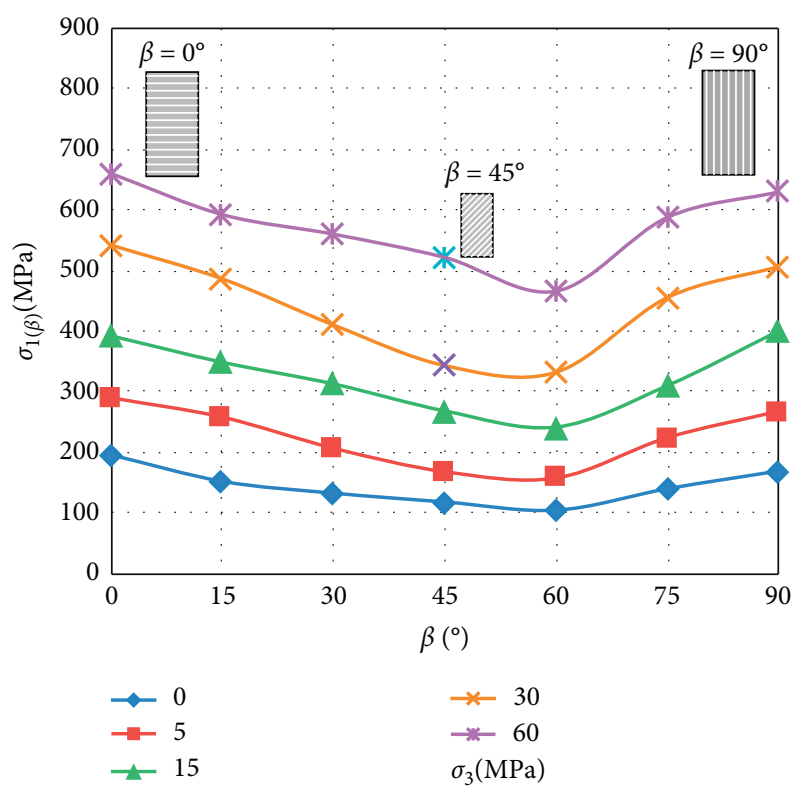

(a)

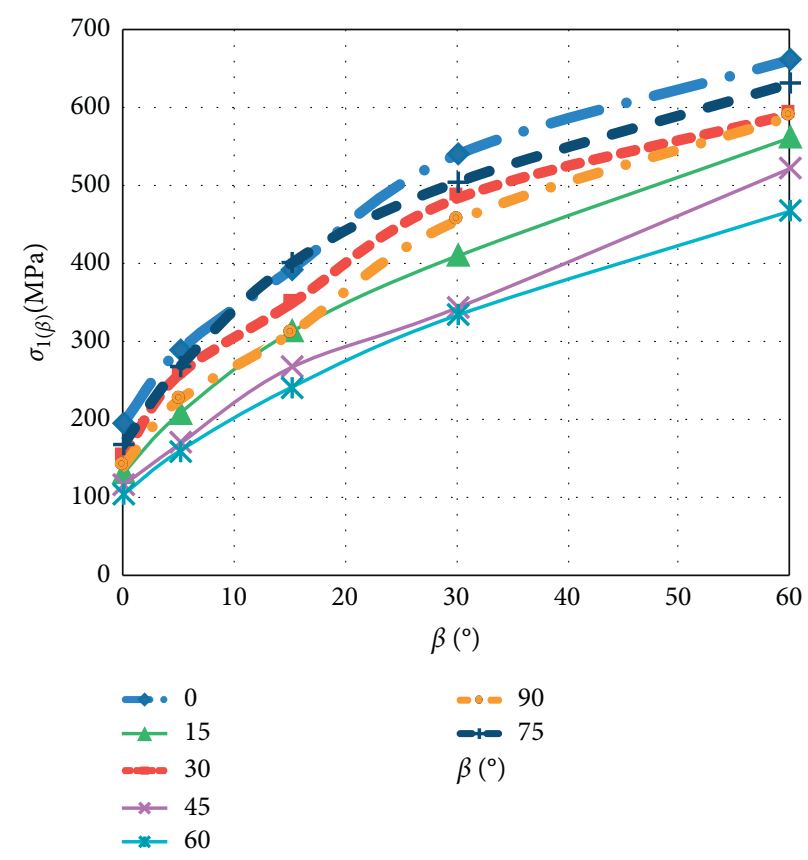

(b)

FIgURE 1: (a) Variation of strength with orientation of bedding. (b) Nonlinear strength behavior (data from [5]).

widely used for anisotropic rocks. Several experiments have been conducted to evaluate the applicability of Jaeger's theory [9-11]. The results also showed that the elastic moduli of layered rocks are transversely isotropic [12]. The ubiquitous joint model of Flac ${ }^{3 \mathrm{D}}$ [13] uses the Jaeger criterion as a plasticity function, and it is widely used in the numerical simulation of jointed rock mass. The authors of [14] conducted quantitative and qualitative syntheses on different failure criteria. However, the anisotropic criterion ideal for rock engineering has not yet been determined.

Therefore, this paper (1) firstly provides a review of the failure criteria for anisotropic rocks among which four representative criteria are selected. (2) The different fitting methods for rock parameters of different strength criteria are designed. A database containing 282 UCS and 1,273 triaxial compression strength from the literature is compiled. (3) Quantitative evaluation of two conditions is conducted that constitutes only a part of the available data because obtaining high-quality data in the engineering field is challenging. The influence of confining pressure on the predictive ability of different criteria is studied. (4) Finally, an open-pit mine slope and a hydropower station underground powerhouse are used as engineering examples to compare the application effects and prediction accuracy of different failure criteria.

\section{Review of Failure Criteria of Anisotropic Rocks}

2.1. Classification of Failure Criteria. Several failure criteria for anisotropic-layered rocks have been developed, which can be classified into three groups [14]: (1) mathematical approach [15-17]; (2) empirical approach [3, 18-21]; and (3) discontinuous criteria $[9,22]$. The former two are classified as continuous and the latter is classified as discontinuous. Table 1 presents the classification. In the first group, most material parameters have no direct physical meaning and lack experimental validation. Therefore, they have not been widely used in engineering practice. Ambrose [59] showed that Pariseau's criterion is the most commonly used mathematical model. In the second group, the friction angle is generally assumed constant, and cohesion (or material parameters related to cohesion) is orientation-specific. Most equations are easily developed or modified from MC and are easy to use. The third group of criteria is developed based on JPW. Contrary to the previous two groups, it is assumed that the rock is composed of matrix and joint, and it is the lower strength between them that play a controlling role in the system. This hypothesis is widely used in engineering because of its clear physical meaning and high-precision strength prediction.

\subsection{Typical Failure Criteria}

2.2.1. Pariseau's Criterion. Pariseau [16] presented a failure criterion for anisotropic geological media. The primary contribution of Pariseau is the modification of Hill's yield theory [26] to account for the yield of geomaterials under hydrostatic stress. The material constants can be reduced to six in the case of transversely isotropic materials and is expressed by 
TABLE 1: Classification of anisotropic failure criteria.

\begin{tabular}{|c|c|c|}
\hline \multicolumn{2}{|c|}{ Continuous criteria } & \multirow{2}{*}{ Discontinuous criteria } \\
\hline Mathematical approach & Empirical approach & \\
\hline Von Mises [23] & Casagrande \& Carrillo [24] & Jaeger $[8,25]$ \\
\hline Hill [26] & Jaeger variable shear $[8]$ & Walsh \& Brace [9] \\
\hline Olszak and Urbanowicz [27] & McLamore and Gray [28] & Hoek [29] \\
\hline Goldenblat [30] & Ramamurth,Rao and Singh [22] & Hoek and Brown [31] \\
\hline Goldenblat and Kopnov [32] & Ashour [33] & Murrel [34] \\
\hline Hoffman [15] & Zhao,Liu and Qi [35] & Barron $[36]$ \\
\hline Pariseau [16] & Singh,et al. [37] & Ladanyi and Archambault [38] \\
\hline Tsai and Wu [17] & Tien, et al. [3] & Bieniawski [39] \\
\hline Boehler [40] & Colak K, Unlu T.* [41] & Smith and Cheatham [42] \\
\hline Dafalias $[43,44]$ & Tiwari and Rao (2007) & Yoshinaka and Yamabe [45] \\
\hline Allirot and Boehler [46] & Saroglou and Tsiambaos [18] & Duveau et al. [14] \\
\hline Nova and Sacchi [40] & Zhang and Zhu [19] & Pei $[47]$ \\
\hline Nova $[48]$ & Saroglou H, Tsiambaos G.[18] & Zhang [49] \\
\hline Boehler and Raclin [50] & Lee Y K, Pietruszczak S.* $\left.{ }^{*} 51\right]$ & \\
\hline Raclin [52] & Lee, Pietruszczak and Choi [20] & \\
\hline Kaar et al. [53] & Yang $\mathrm{Xu}$, et al.* & \\
\hline \multicolumn{3}{|c|}{ Cazacu and Cristescu [54] } \\
\hline \multicolumn{3}{|c|}{ Kusabuka, Takeda and Kojo [55] } \\
\hline \multicolumn{3}{|c|}{ Pietruszczak et al. [56] } \\
\hline \multicolumn{3}{|l|}{ Chen and Yang* [57] } \\
\hline \multicolumn{3}{|l|}{ Lee and Pietruszczak [51] } \\
\hline Mroz and Maciejewski [58] & & \\
\hline
\end{tabular}

${ }^{*}$ Criteria added since $[14,59]$

$$
\left[F\left(\sigma_{x x}-\sigma_{y y}\right)^{2}+G\left\{\left(\sigma_{x x}-\sigma_{z z}\right)^{2}+\left(\sigma_{z z}-\sigma_{y y}\right)^{2}\right\}+(2 G+4 F) \sigma_{x y}^{2}+M\left(\sigma_{z x}^{2}+\sigma_{z y}^{2}\right)\right]^{n / 2}-\left[U \sigma_{z z}+V\left(\sigma_{x x}+\sigma_{y y}\right)\right]=1
$$

Pariseau's criterion is an extension of the Drucker-Prager yield criterion when $n=1$ and is equivalent to the Hoffman criterion when $n=2$. Thus, Hoffman criterion is a specific case of Pariseau's criterion. The two cases of discussion are $n=1$ and $n=2$. Equation 1 is reduced to the following by using stress coordinate transformation when applied to conventional triaxial $\left(\sigma_{1}>\sigma_{2}=\sigma_{3}\right)$ :

$$
\begin{aligned}
\sigma_{1}-\sigma_{3} & =\frac{1+\sigma_{3}(U+2 V)}{\left(F \sin ^{4} \beta+G\left(\cos ^{4} \beta+\cos ^{2} 2 \beta\right)+0.25 M \sin ^{2} 2 \beta\right)^{0.5}-\left(U \cos ^{2} \beta+V \sin ^{2} \beta\right)}, \\
\sigma_{1}-\sigma_{3} & =\frac{\left(U \cos ^{2} \beta+V \sin ^{2} \beta\right)+\sqrt{\left(U \cos ^{2} \beta+V \sin ^{2} \beta\right)^{2}+4\left(F \sin ^{4} \beta+G\left(\cos ^{4} \beta+\cos ^{2} 2 \beta\right)+0.25 M \sin ^{2} 2 \beta\right)\left(1+\sigma_{3}(U+2 V)\right)}}{2\left(F \sin ^{4} \beta+G\left(\cos ^{4} \beta+\cos ^{2} 2 \beta\right)+0.25 M \sin ^{2} 2 \beta\right)} .
\end{aligned}
$$

Earlier studies show that the five material parameters can be expressed in terms of uniaxial compression, tension, and pure shear strength when $n=1$ [60]. Hoffman [15] proposed a similar deduction and obtained the expression when $n=2$ (Table 2).

In this study, the strength parameters of layered rocks are obtained by experiments, where $X_{\mathrm{t}}=4.45 \mathrm{MPa}$, $X_{\mathrm{c}}=134.37 \mathrm{MPa}, Y_{t}=12.70 \mathrm{MPa}, Y_{\mathrm{c}}=119.10 \mathrm{MPa}$, and $S=3.4 \mathrm{MPa}$; the detailed experimental procedure has been explained by Wang [61]. By considering the strength parameters given in Table 2 and employing equations (2) and (3), the uniaxial compressive strength can be predicted, as shown in Figure 2.
The results show a slight deviation between the predicted values and the mean values when $n=1$ or 2 . Particularly, the predicted minimum strength is lower than the actual value when $\beta=60^{\circ}$. It is difficult to obtain accurate uniaxial compression, tension, and pure shear strength owing to the dispersion of strength of rock. Thus, Table 2 is not recommended to predict compressive strength; it cannot verify the prediction ability of triaxial strength because of the lack of conventional triaxial test data. Next, the material parameters in equations (2) and (3) are solved by optimizing different values of triaxial strength from the literature. The compressive strength prediction ability of Pariseau's criterion is evaluated. 
TABle 2: The material coefficients of Pariseau's criteria.

\begin{tabular}{cccccc}
\hline$n$ & $F$ & $G$ & $M$ & $U$ & $V$ \\
\hline 1 & $\left(Y_{t}^{-1}+Y_{c}^{-1}\right)^{2} / 4-G$ & $\left(X_{t}^{-1}+X_{c}^{-1}\right)^{2} / 8$ & $S^{-2}$ & $\left(X_{t}^{-1}-X_{c}^{-1}\right) / 2$ & $X_{t}^{-1}-X_{c}^{-1}$ \\
2 & $\left(Y_{t} Y_{c}\right)^{-1}-\left(X_{t} X_{c}\right)^{-1} / 2$ & $\left(X_{t} X_{c}\right)^{-1} / 2$ & $S^{-2}$ & $\left(Y_{t}^{-1}-Y_{c}^{-1}\right) / 2$ \\
\hline
\end{tabular}

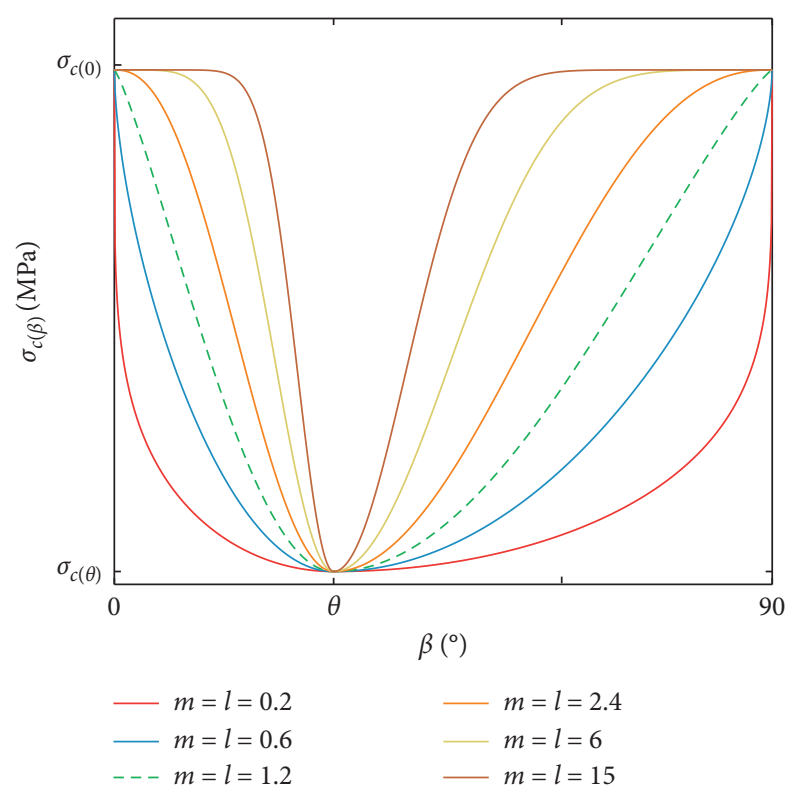

FIgURE 2: Predicted of Pariseau's criterion vs. experimental UCS.

2.2.2. Liner and Nonlinear Empirical Equations. An empirical equation to predict the uniaxial compressive strength for anisotropic rocks was proposed by Xu et al. [21] based on Jaeger's model $[8,10]$, denoted by equation (4). With an increase in $m$ and $l$, the layered rocks gradually change from U-type to shoulder-type anisotropy (see Figure 3). Therefore, equation (4) can predict the uniaxial compressive strength of various layered rocks.

$$
\sigma_{c(\beta)}= \begin{cases}\sigma_{c\left(0^{\circ}\right)}-\left(\sigma_{c\left(0^{\circ}\right)}-\sigma_{c(\theta)}\right)\left[\sin \left(\frac{\beta}{\theta} \cdot 90^{\circ}\right)\right]^{m}, & \text { if } 0^{\circ} \leq \beta \leq \theta, \\ \sigma_{c\left(90^{\circ}\right)}-\left(\sigma_{c\left(90^{\circ}\right)}-\sigma_{c(\theta)}\right)\left[\cos \left(\frac{\beta-\theta}{90^{\circ}-\theta} \cdot 90^{\circ}\right)\right]^{l}, & \text { if } \theta<\beta \leq 90^{\circ} .\end{cases}
$$

From equation (4) and the Mohr-Coulomb criterion, a linear empirical equation for compressive strength of layered rocks is expressed as

$$
\sigma_{1}-\sigma_{3}=\sigma_{c(\beta)}+\frac{2 \sin \phi}{1-\sin \phi} \sigma_{3}
$$

As the confining pressure increases, the rate of increase in strength decreases, and the failure mode tends to change from brittle to ductile. A nonlinear failure criterion for anisotropic rocks was proposed by Singh et al. [5] based on the critical state concept, expressed by equation (6). From equations (4) and (6), a nonlinear empirical equation for 


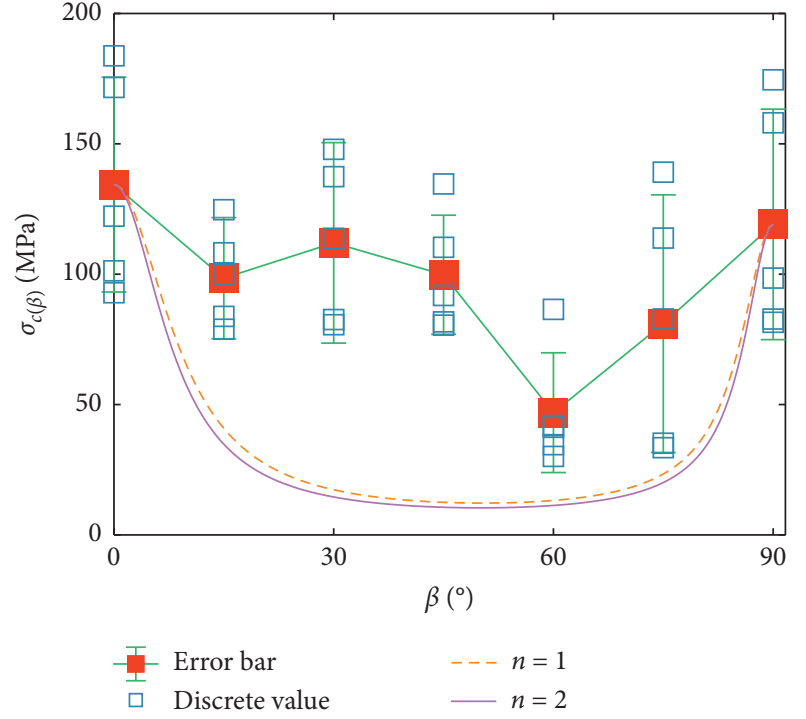

FIGURE 3: Variations of UCS with orientation angle $\beta$ and various values of $m$ and $l$.

compressive strength of layered rocks can be obtained. When the confining pressure is greater than $\sigma_{c r t}$, the differential stress tends to be constant (Figure 4). Therefore, the model can be regarded as a modification of the Mohr-Coulomb criterion.

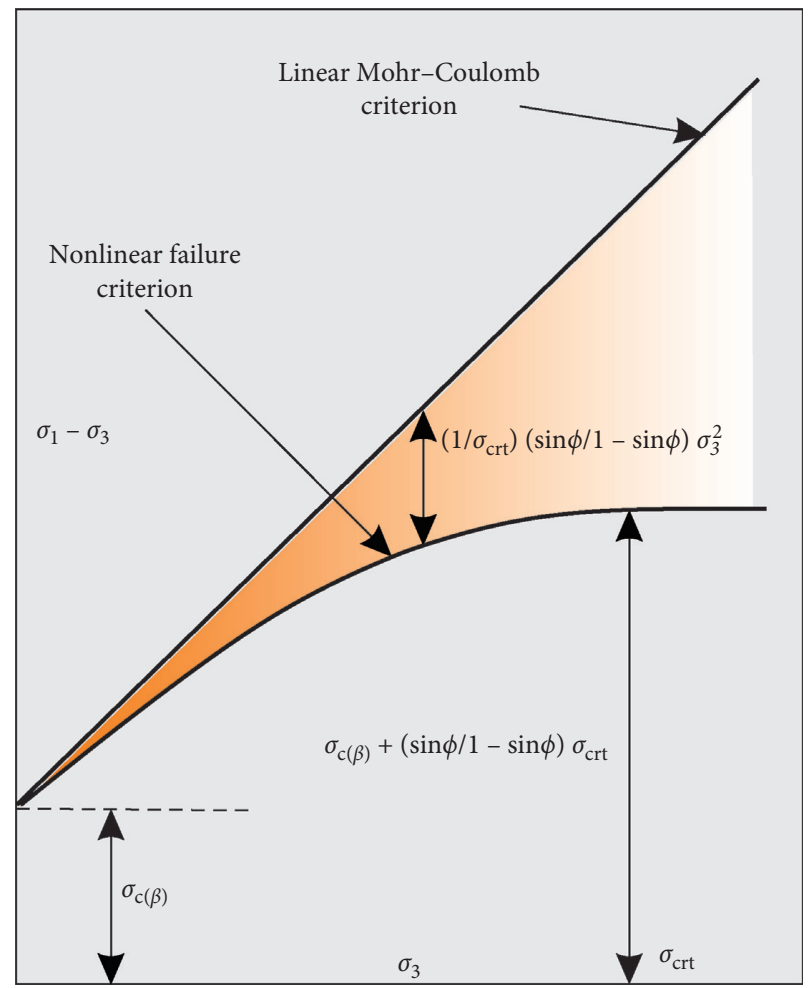

Figure 4: Nonlinear failure criterion proposed by Singh et al. [5].

$$
\sigma_{1}-\sigma_{3}= \begin{cases}\sigma_{c(\beta)}+\frac{2 \sin \phi}{1-\sin \phi} \sigma_{3}-\frac{1}{\sigma_{\mathrm{crt}}} \frac{\sin \phi}{1-\sin \phi} \sigma_{3}^{2}, & \text { if } 0 \leq \sigma_{3} \leq \sigma_{\mathrm{crt}}, \\ \sigma_{c(\beta)}+\frac{\sin \phi}{1-\sin \phi} \sigma_{\mathrm{crt}}, & \text { if } \sigma_{3}>\sigma_{\mathrm{crt}} .\end{cases}
$$

2.2.3. Jaeger's Plane of Weakness Model. Jaeger [8] introduced JPW, which is the start point of the discontinuous method. The rock matrix and weakness planes are both described by Mohr and Coulomb. However, there are diffident values in cohesion and friction for rock matrix and weakness planes. Thus, the failure criterion could be given as

$\sigma_{1}-\sigma_{3}=\min \left(\frac{2 \sin \phi_{\mathrm{m}}}{1-\sin \phi_{m}} \sigma_{3}+\frac{2 c_{\mathrm{m}} \cos \phi_{m}}{1-\sin \phi_{m}}, \frac{2\left(c_{w}+\sigma_{3} \tan \theta_{w}\right)}{\left(1-\tan \theta_{w} \cot \beta\right) \sin 2 \beta}\right)$.

\section{Fitting Parameters of Failure Criteria}

3.1. Parameters of Evaluation. For assessing the predictive accuracy, regression coefficient $\left(R^{2}\right)$ and mean absolute percentage error (MAPE) are used for quantitative evaluation. The larger the $R^{2}$ and the smaller the MAPE, the better the predictive accuracy:

$$
\begin{gathered}
R^{2}=1-\frac{\sum\left(\sigma_{c, \exp }-\sigma_{c, \text { pre }}\right)^{2}}{\sum\left(\sigma_{c, \exp }-\sigma_{c, a v}\right)^{2}}, \\
\text { MAPE }=\frac{\sum\left|\sigma_{c, \exp }-\sigma_{c, \text { pre }} / \sigma_{c, \exp }\right|}{N} \times 100 \% .
\end{gathered}
$$

3.2. Algorithm of Fit. The dispersion in the macroscopic strength of rocks is large due to the heterogeneity, and it is difficult to obtain an accurate strength variation curve. Therefore, a vast amount of experimental data is essential. To a certain extent, the above strength criteria can be regarded as basic formulas for describing the strength law of layered rocks. The unknown coefficients in equations (2)-(7) can be fitted by the optimization algorithm based on sufficient experimental data. The uniaxial and triaxial strength data in this study are derived from the literature. 
Each strength criterion is regarded as $\sigma_{1}=\sigma_{1}\left(\sigma_{3}, \beta\right)$ in which Pariseau's criterion is a continuous nonlinear function and LEE, NLEE, and JPW are linear or nonlinear piecewise functions. The optimization algorithms for these types of functions are different. The continuous nonlinear function can use the nonlinear least squares method. In this study, a trust-region algorithm is used to solve the material parameters $F, G, M, U$, and $V$ with the curve fitting tool in MATLAB. The linear or nonlinear piecewise functions cannot use the least squares method. However, most material parameters of these functions have clear and direct physical meaning. The range of parameters can be determined by engineering experience. For example, the friction angle of rocks is generally in the range of $10-50^{\circ}$. Therefore, it is feasible to use the traversing method for this type of problem. Through searching all combinations of material parameters with a fixed sampling interval, the best fit parameters are found. Then, the range and interval $\boldsymbol{d}$ is refined, and the search is repeated. The abovementioned process is repeated iteratively until the optimal strength parameters of the rocks are determined. The flow chart of the fit algorithm is show in Figure 5.

Taking the triaxial data of bedding sandstone and data from Deng et al. [62] as examples, Table 3 details the results of the parameters. The comparison between the predicted strength and the measured data is shown in Figures 6(a)$6(\mathrm{f})$. The results show that $R^{2}$ of Pariseau $(n=(2)$ is lower than 0.7 , and MAPE is higher than $20 \%$. Thus, the prediction precision is poor. The goodness of fit for the other failure criteria is greater than 0.85 , and MAPE is less than $16 \%$. This shows that the material parameters in different failure criteria can be determined by the nonlinear least squares method and the traversing method, and the prediction precision is relatively high with sufficient triaxial data. This study solves the problem of low prediction precision of Pariseau $\left(n=(2)\right.$ by generating $10^{4}$ random initial values. As shown in Figure 6(c), the prediction precision is low which excludes the influence of initial values on the optimal solution. This implies that the criterion may not be applicable to layered rocks when $n=2$. Further verification is made in the following sections.

3.3. Database. In this study, the triaxial strength database of transversely isotropic rocks by Singh et al. [5] is used, and five other rock types $[14,59,62,63]$ are added to the database. The compiled database comprises triaxial test results on 43 rock types with a total number of 282 UCS and 1,273 triaxial tests. The complete database and the complete programs are available on GitHub.

\section{Fitting Results}

4.1. All Data Available. Assuming all databases are available, the predicted $\sigma_{1}$ can be obtained by the aforementioned method. Figure 7 shows the plot of 1555 predicted values against the experimental values. The figure shows that the predicted value of Pariseau $(n=2)$ is higher than the test value in total (Figure $7(\mathrm{~b})$ ), which further proves that
Hoffman is not suitable for layered rocks. Therefore, it is not further evaluated. The other criteria show a very high $R^{2}$ value of approximately 0.97 , and MAPEs are between $11 \%$ and $16 \%$. The predictions are good for the four criteria when all data are available. Combining the two indexes, $R^{2}$ and $M A P E$, the precision of each failure criterion is NLEE $>$ LEE $>$ Pariseau $(n=1)>$ JPW .

The reasons for the difference in prediction accuracy are as follows: (1) the relationship between $\sigma_{1}$ and $\sigma_{3}$ of Pariseau $(n=1)$, LEE, and JPW criteria is linear, whereas the relationship between $\sigma_{1}$ and $\sigma_{3}$ of Pariseau $(n=2)$ and NLEE criteria is nonlinear when $\beta$ is constant. However, Pariseau $(n=2)$ is an exponential function as $\sigma_{1}\left(\sigma_{3}, \sigma_{3}{ }^{0.5}\right)$. This form does not conform to the nonlinear strength characteristics of rocks under high pressure. Therefore, the fitting results have larger errors. On the contrary, the NLEE has a better prediction because it is further modified based on the LEE relationship, which conforms to the true law of rock strength varying with confining pressure; (2) JPW is classified as piecewise functions at the same time, as shown in Figure 6(f). It is difficult to accurately describe the strength of $\beta$ in the transition stage at approximately $30^{\circ}$. Hence, the prediction accuracy of the JPW criterion is lower than that of the empirical equation and Pariseau $(n=1)$; (3) the numbers of material parameters of each criterion selected in the study are four (JPW), five (Pariseau $n=1$ and $n=2$ ), six (LEE), and seven (NLEE). The prediction accuracy is proportional to the number of material parameters. The more the number of material parameters, the higher the prediction accuracy (except when Pariseau $n=2$ ).

The study [5] shows that the critical confining pressure for inherently anisotropic rocks may be taken approximately equal to $1.25 \sigma_{\text {cmax }}$. Therefore, all experimental data are divided into two types, namely, medium-low confining pressure and high confining pressure, and expressed in blue and red in Figures 7 and 8, respectively. The predictive accuracy of different criteria under the two types is further analyzed in the following sections.

4.2. Part of Data Available. Owing to the high cost and time of triaxial experiment, the failure criteria should exhibit good prediction accuracy even when only a part of data is available. It should be noted that (1) the internal friction angle cannot be determined only by uniaxial data, so the parts of triaxial data are necessary; (2) the strength values of the three angles $\left(0^{\circ}, 90^{\circ}, \theta\right)$ of $\beta$ are the key points of the strength curve of layered rocks, and increasing the strength data of other inclined rock can further improve the predicted accuracy; (3) since Pariseau's criterion is solved by the nonlinear least squares method, the minimum data cannot be less than six. Therefore, three different conditions for the availability of data, which might offer the lowest accuracy in practical application, are considered in this paper. These conditions are as follows: (1) all UCS and a set of triaxial data are available; $(2) \sigma_{\mathrm{c}\left(0^{\circ}\right)}, \sigma_{\mathrm{c}\left(90^{\circ}\right)}, \sigma_{\mathrm{c}(\theta)}$, and USC of an arbitrary $\beta$ and a set of triaxial data with the corresponding $\beta$ are available; (3) $\sigma_{\mathrm{c}\left(0^{\circ}\right)}, \sigma_{\mathrm{c}\left(90^{\circ}\right)}, \sigma_{c(\theta)}$, and a set of triaxial data with the corresponding $\beta$ are available. As shown in Figure 8, 


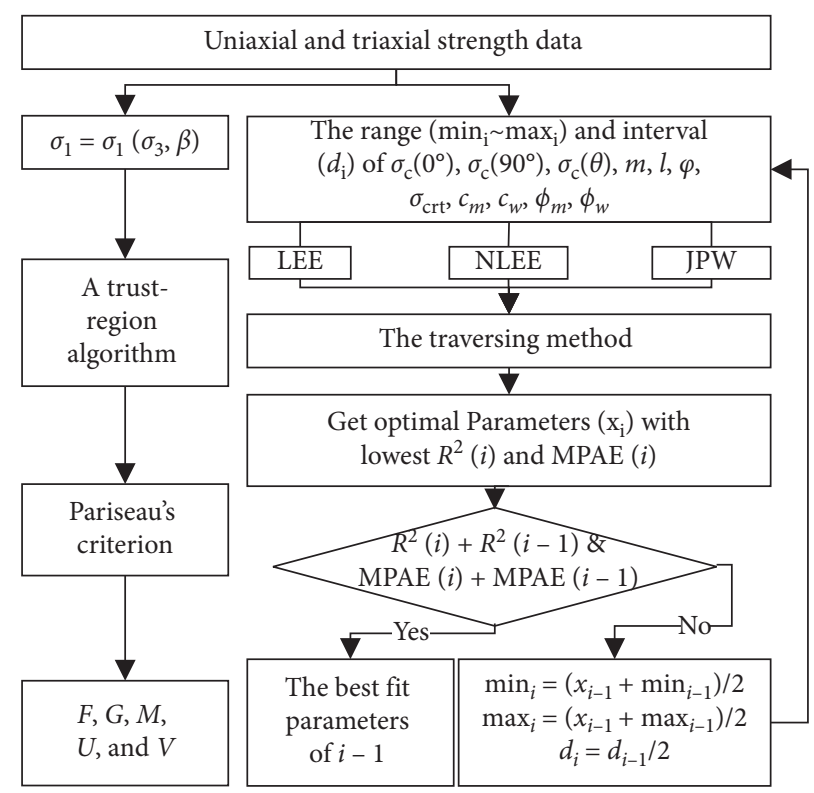

Figure 5: Flow Chart of algorithm of fit.

TABLE 3: The material coefficients of different criteria.

\begin{tabular}{|c|c|c|c|c|c|c|c|c|c|}
\hline Pariseau & $R^{2}$ & MAPE & $F$ & $G$ & $U$ & $V$ & $W$ & - & - \\
\hline$n=1$ & 0.977 & 8.17 & 0.000055 & 0.003972 & 0.022213 & 0.071354 & 0.044727 & & \\
\hline$n=2$ & 0.671 & 24.18 & 0.011329 & 0.000587 & 0.016563 & 0.00924 & 1.33743 & & \\
\hline Empirical equation & $R^{2}$ & MAPE & $\sigma_{c(0)}$ & $\sigma_{c(90)}$ & $\sigma_{c(\theta)}$ & $\phi$ & $m$ & $l$ & $\sigma_{\mathrm{crt}}$ \\
\hline Linear & 0.996 & 3.98 & $60.68 \mathrm{MPa}$ & $66.73 \mathrm{MPa}$ & $18.82 \mathrm{MPa}$ & $53.19^{\circ}$ & 5.83 & 2.67 & - \\
\hline Nonlinear & 0.997 & 3.42 & $58.88 \mathrm{MPa}$ & $64.72 \mathrm{MPa}$ & $17.19 \mathrm{MPa}$ & $54.26^{\circ}$ & 6.18 & 2.33 & $58.69 \mathrm{MPa}$ \\
\hline JPW & $\begin{array}{c}R^{2} \\
0.857\end{array}$ & $\begin{array}{c}\text { MAPE (\%) } \\
15.83\end{array}$ & $\begin{array}{c}C_{m} \\
10.79 \mathrm{MPa}\end{array}$ & $\begin{array}{c}\phi_{m} \\
52.17^{\circ}\end{array}$ & $\begin{array}{c}C_{w} \\
5.74 \mathrm{MPa}\end{array}$ & $\begin{array}{c}\phi_{w} \\
48.51^{\circ}\end{array}$ & - & - & - \\
\hline
\end{tabular}

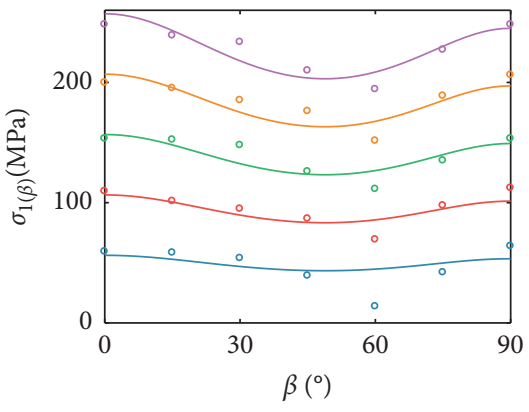

Predictive value: Distrate value:

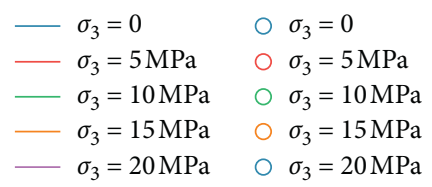

(a)

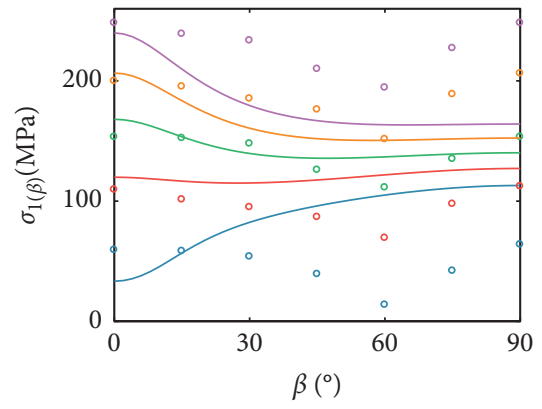

Predictive value: Distrate value:

$\begin{array}{rlrl}-\sigma_{3} & =0 & & \bigcirc \sigma_{3}=0 \\ \sigma_{3}=5 \mathrm{MPa} & & \bigcirc \sigma_{3}=5 \mathrm{MPa} \\ \sigma_{3} & =10 \mathrm{MPa} & & \bigcirc \sigma_{3}=10 \mathrm{MPa} \\ -\sigma_{3} & =15 \mathrm{MPa} & & \bigcirc \sigma_{3}=15 \mathrm{MPa} \\ -\sigma_{3} & =20 \mathrm{MPa} & & \bigcirc \sigma_{3}=20 \mathrm{MPa}\end{array}$

(b)

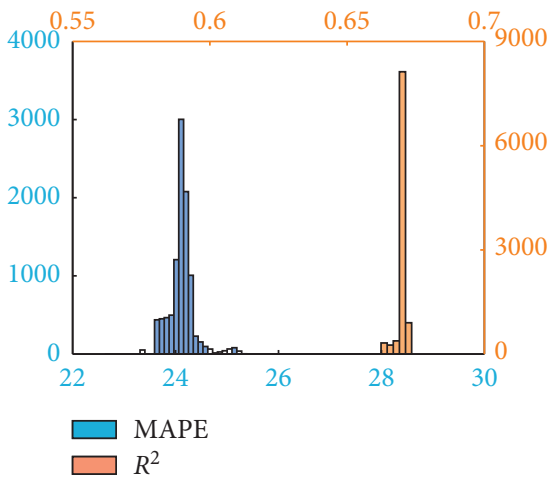

$\square R^{2}$

FIgURe 6: Continued. 


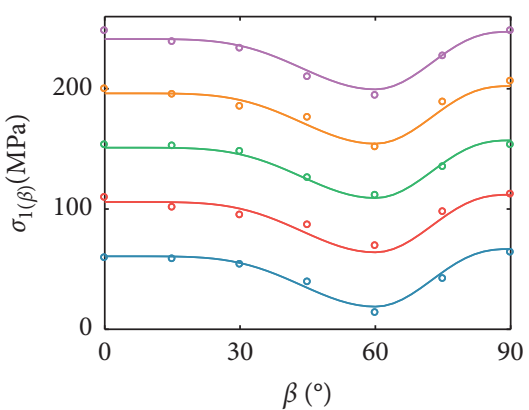

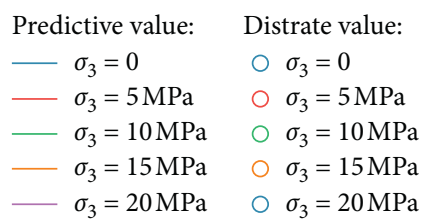

(d)

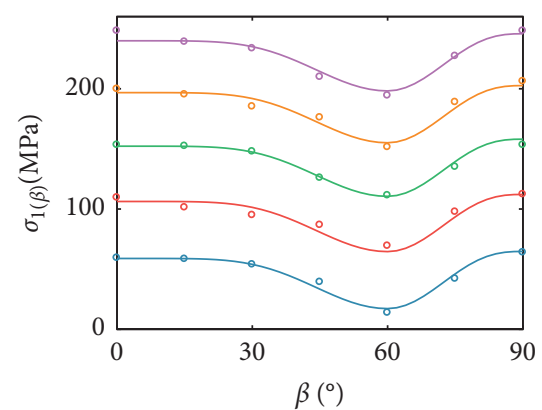

Predictive value:

$\begin{array}{lll}-\sigma_{3}=0 & & \sigma_{3}=0 \\ -\sigma_{3}=5 \mathrm{MPa} & & \bigcirc \sigma_{3}=5 \mathrm{MPa} \\ -\sigma_{3}=10 \mathrm{MPa} & & \bigcirc \sigma_{3}=10 \mathrm{MPa} \\ -\sigma_{3}=15 \mathrm{MPa} & & \bigcirc \sigma_{3}=15 \mathrm{MPa} \\ -\sigma_{3}=20 \mathrm{MPa} & & \bigcirc \sigma_{3}=20 \mathrm{MPa}\end{array}$

(e)

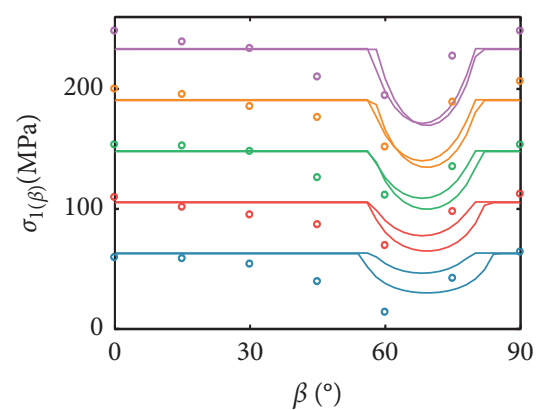

Predictive value:

$-\sigma_{3}=0$

$-\sigma_{3}=5 \mathrm{MPa}$

Distrate value:

$-\sigma_{3}=10 \mathrm{MPa}$

$\bigcirc \sigma_{3}=0$

$-\sigma_{3}=15 \mathrm{MPa}$

$\circ \sigma_{3}=5 \mathrm{MPa}$

$\circ \sigma_{3}=10 \mathrm{MPa}$

$\circ \sigma_{3}=15 \mathrm{MPa}$

$-\sigma_{3}=20 \mathrm{MPa} \bigcirc \sigma_{3}=20 \mathrm{MPa}$

(f)

Figure 6: Predicted vs. experimental strength of sandstone (data from [62]). (a) Pariseau $n=1$. (b) Pariseau $n=2$. (c) Random starting value. (d) LEE. (e) NLEE. (f) JPW.

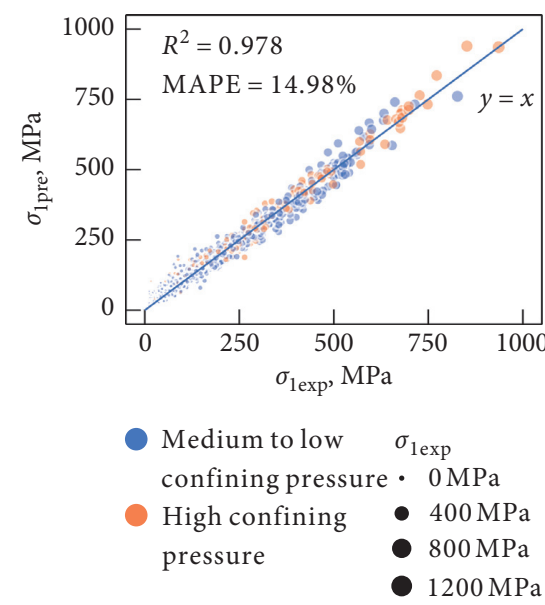

(a)

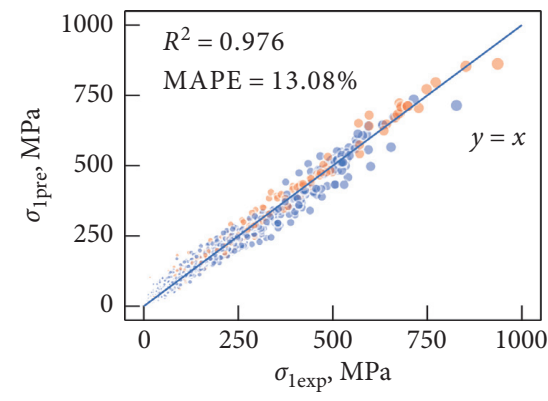

Medium to low $\sigma_{\text {lexp }}$ confining pressure $\cdot 0 \mathrm{MPa}$

High confining pressure

- $400 \mathrm{MPa}$

- $800 \mathrm{MPa}$

- $1200 \mathrm{MPa}$

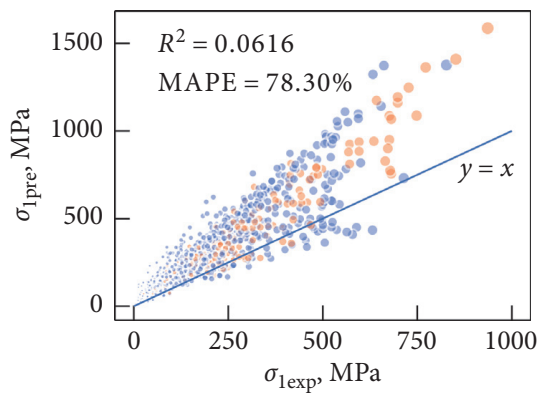

Medium to low $\sigma_{1 \exp }$ confining pressure $\cdot 0 \mathrm{MPa}$

High confining pressure

- $400 \mathrm{MPa}$

- $800 \mathrm{MPa}$

- $1200 \mathrm{MPa}$

(b)

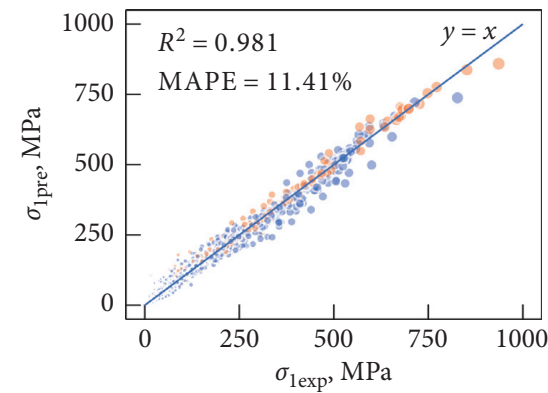

Medium to low $\sigma_{\text {lexp }}$ confining pressure $\cdot 0 \mathrm{MPa}$

High confining pressure

- $400 \mathrm{MPa}$

- $800 \mathrm{MPa}$

- $1200 \mathrm{MPa}$

(c)

(d)

FIgURE 7: Continued. 


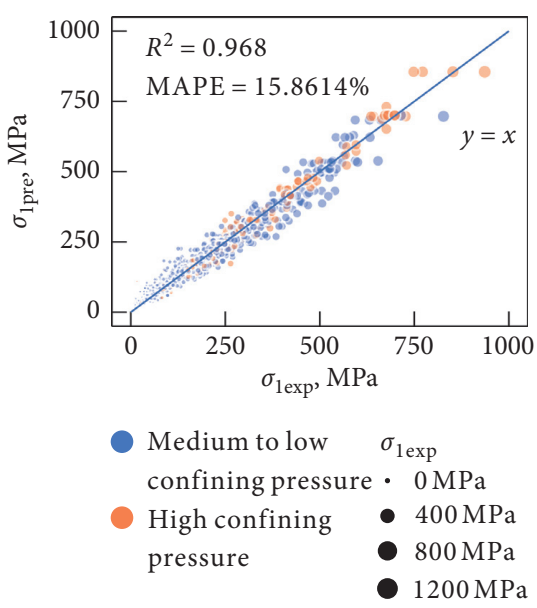

(e)

Figure 7: Predicted vs. experimental $\sigma_{1}$ values when all data available. (a) Pariseau $n=1$. (b) Pariseau $n=2$. (c) Linear empirical equation. (d) Nonlinear empirical equation. (e) JPW.

obtaining more experimental data will lead to higher prediction accuracy if conditions permit.

The results show that the predicted value of Pariseau's criterion $(n=1)$ is greater than the test value in total, its $R^{2}$ value is below 0.1 , and MAPE fluctuates by $26 \%$. The prediction precision of all criteria tends to decrease with a decrease in the available data. By combining $R^{2}$ and MAPE under the three conditions, the prediction accuracy of each failure criterion becomes NLEE $>($ JPW $\approx$ LEE $)>$ Pariseau.

Considering all available data, some changes can be seen in the prediction accuracy. Specifically, the goodness of fit of Pariseau's $(n=1)$ criterion is much lower as compared to the other three criteria. The reason may be that Pariseau's criterion adopts an unconstrained optimization method. The parameters related to the friction angle $(U$ and $V)$ may be too large for the strength on high pressure when only UCS and a set of triaxial data are available. Therefore, some of the predicted strengths are much higher as compared to the test values with an increase in the confining pressure. For instance, some of the predicted values in Figure 8 are as high as $2000 \mathrm{MPa}$, whereas the experimental maximum values are only $1000 \mathrm{MPa}$. The other strength criteria are optimized by applying traversing method, and the parameters of the friction angle are limited within a reasonable range. Therefore, no excessive deviation will occur. Considering the nonlinear characteristics of rock mass strength, the overall prediction accuracy of NLEE is considered to be the best. However, the fitting of $m$ and $l$ parameters of NLEE and LEE lacks sufficient data because only the key angle data can be obtained. Therefore, the prediction accuracy of LEE is not higher than that of JPW.

4.3. Effect of the Confining Pressure. As shown in Figures 7 and 8 , the predicted value and the test value are compared under the medium to low and high confining pressure. The predicted values are distributed on both sides of $y=x$ when all data are available, and the confining pressure has no obvious impact on the predicted accuracy. Once a part of data is available, NLEE can be compared with LEE (as shown in Figure 8) and the predicted value of the former comes to be closer to the test data under high confining pressure.

As shown in Figure 9, the $R^{2}$ value and MAPE in each case indicate that the empirical equations (LEE and NLEE) have the good predictive accuracy $(M A P E<20 \%)$ under the condition of medium to low confining pressure. Moreover, the predictive accuracy of all criteria tends to decrease under the condition of high confining pressure. Furthermore, JPW is considered the best among all (MAPE <40\%).

The primary reason for the influence of confining pressure on the prediction accuracy is the mechanical characteristics of the nonlinear strength caused by the brittle-ductile transition of layered rock under high confining pressure. Owing to the use of nonlinear strength criterion, the prediction accuracy of NLEE is higher as compared to that of LEE under high confining pressure. MPAE is increased by $10 \%$ without obtaining sufficient data. In this paper, the empirical equations NLEE and LEE are assumed to have the same parameters for the friction angle at any $\beta$. The friction angle parameters are fitted with UCS and triaxial data under low confining pressure when part of data is available. The parameters of the friction angle are overestimated when confining pressure is higher as compared to the critical value $\sigma_{\mathrm{crt}}$, which indicates that the overall predicted strength is higher. The friction angles of the rock matrix and joint are both used to control the strength of the layered rocks in JPW. The friction angles of the rock matrix fitted in this database are generally found to be higher as compared to that of the joint, as shown by the fitting result in Table 3. Therefore, the lower friction angle of the joint can 

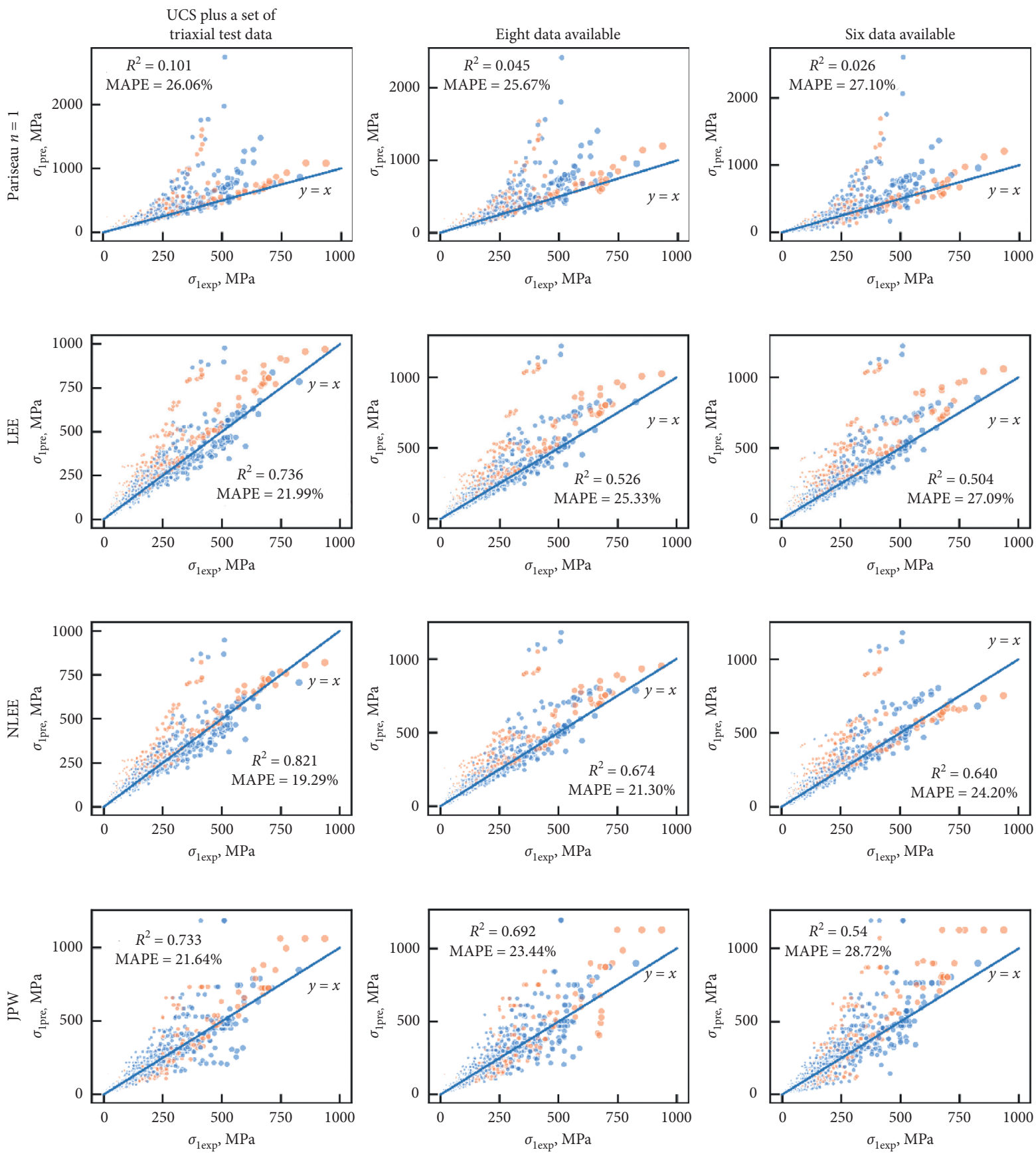

- Medium to low confining pressure

- High confining pressure

FIGURE 8: Predicted vs. experimental $\sigma_{1}$ values when part of data is available.

effectively control the strength under the condition of high confining pressure when $\beta$ is between $30^{\circ}$ and $80^{\circ}$. Consequently, the prediction accuracy of JPW is considered to be the best of all under the condition of high confining pressure.

\section{Engineering Application}

In this section, a shallow open-pit slope and a deep underground powerhouse are considered as engineering cases. The stress field distribution of the surrounding rock is calculated by using the COMSOL multiphysics [64] based on a transversely isotropic elastic constitutive model, and then the value of $\beta$ is calculated. Then, equations (2)-(7) are introduced in the calculation, respectively. Different failure criteria are used to evaluate the damage zone of the surrounding rock in the case of high and low in situ stress. By comparing the actual rock failure, the prediction accuracy in engineering is verified.

5.1. Case 1: Open-Pit Slope. As shown in Figure 10(a), the Yanshan open-pit is located in the Tangshan city in northeastern China, and it is a sedimentary metamorphic 

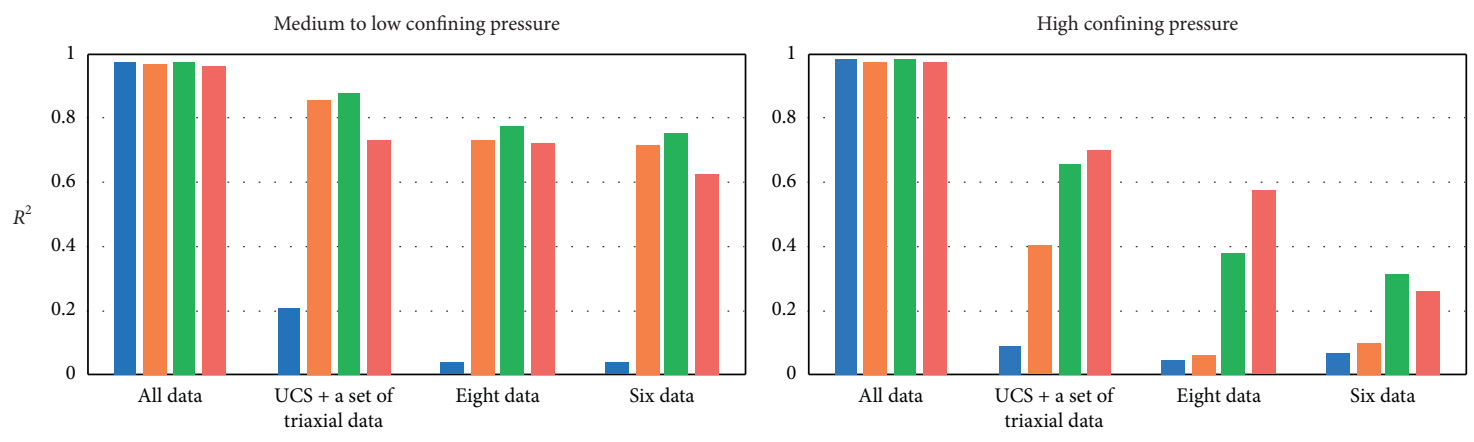

- Pariseau

- Nonlinear empirical

- Pariseau

- Nonlinear empirical

- Linear empirical

- Jpw

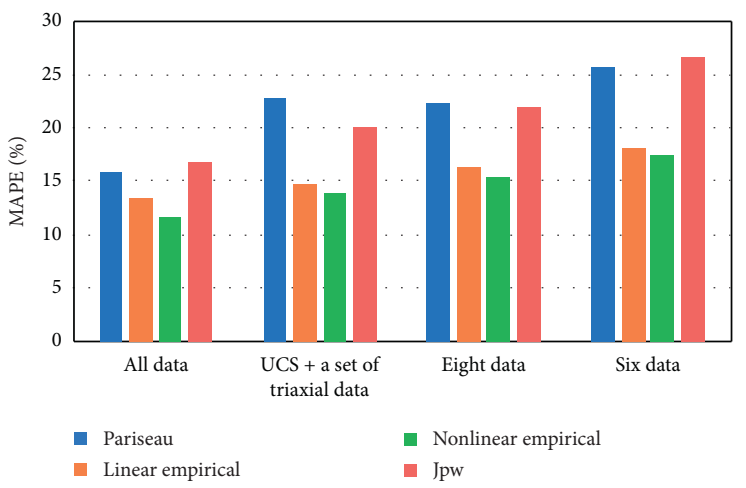

- Jpw

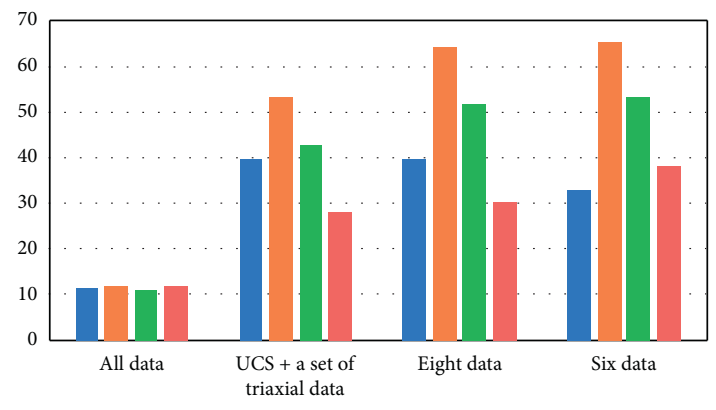

- Pariseau

- Nonlinear empirical

FIGURE 9: The R2 and MPAE of all criteria under different conditions.

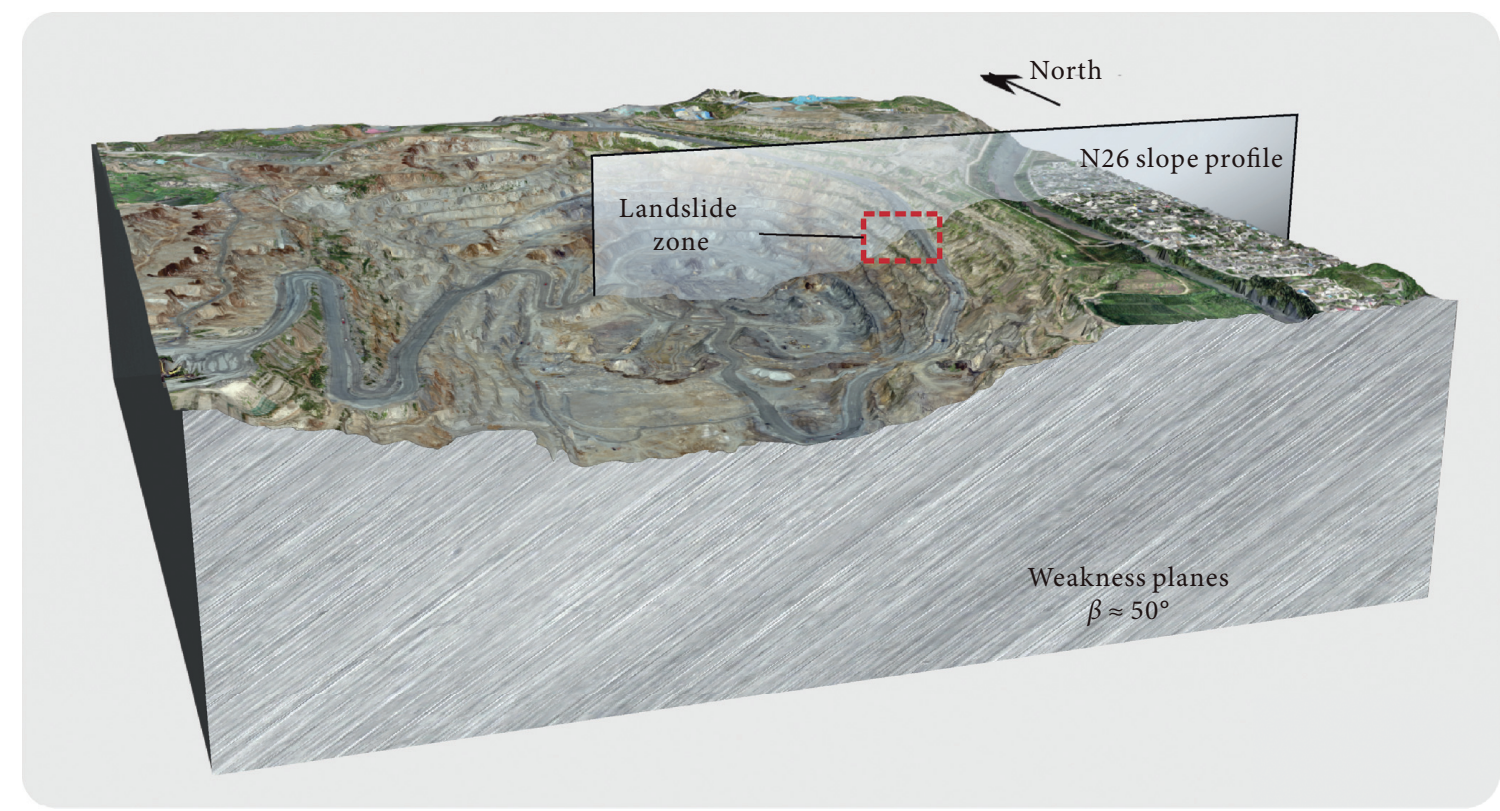

(a)

Figure 10: Continued. 


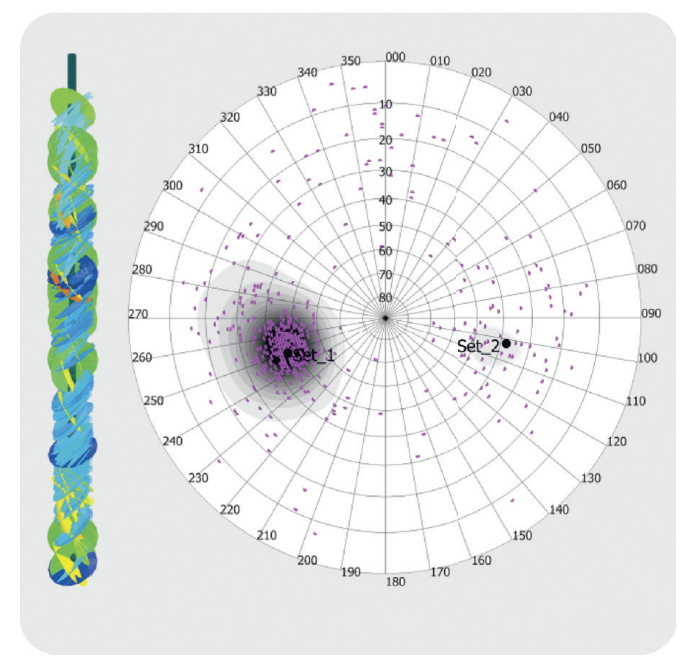

(b)

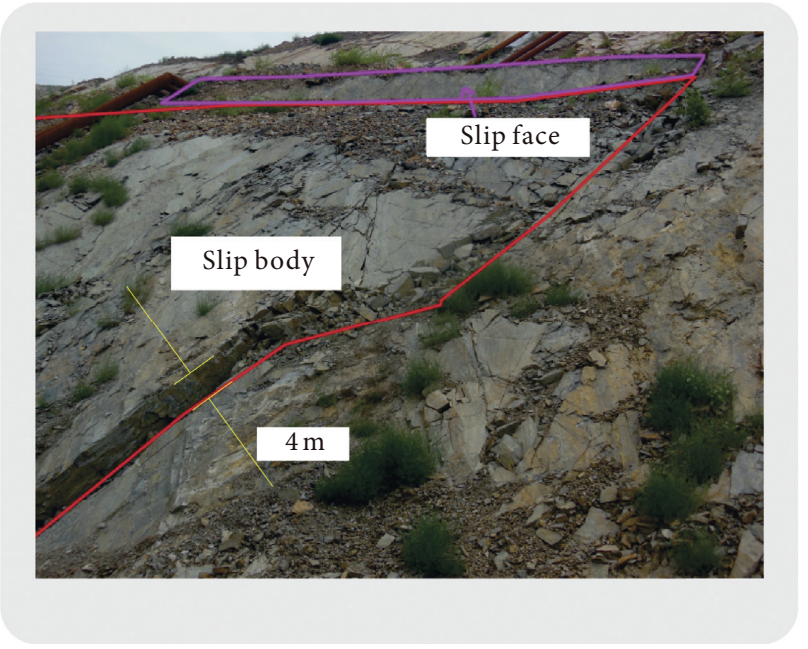

(c)

FIGURE 10: (a) Outcrop of Yanshan open pit and the location of N26 exploration line. (b) Contouring plot of discontinuity orientation. (c) Photo of the planar sliding.

iron mine. As shown in Figure 10(b), structural planes with dip angles of $45^{\circ}-60^{\circ}$ have a percentage of nearly $90 \%$ in the rock mass of the eastern slope based on the borehole TV data. Moreover, two landslide hazards occurred in the eastern slope near the N26 exploration line of the Yanshan open pit on 23 July 2016. Since the average dip angle of weakness planes is less than that of the bench, typical planar sliding was observed as shown in Figure 10(c). Furthermore, the thickness of the slip body is about 4 meters.

For determining mechanical parameters, a detailed experimental procedure and process can be referred to the literature [61]. Mechanical parameters of the equivalent layered rock mass are shown in Table 4. The failure types of the eastern bedding slope are primarily observed in the shallow landslide, and the damage zone is found in the lowstress level. Considering the cohesion and friction angles
TABLE 4: Mechanical parameters of layered rock mass [61].

\begin{tabular}{lcc}
\hline$\beta\left(^{\circ}\right)$ & Cohesive strength $(\mathrm{MPa})$ & Friction angle $\left(^{\circ}\right)$ \\
\hline 0 & 0.27 & 40.91 \\
15 & 0.2 & 35.73 \\
30 & 0.2 & 35.5 \\
45 & 0.19 & 33.85 \\
60 & 0.18 & 32.62 \\
75 & 0.14 & 28.06 \\
90 & 0.22 & 37 \\
\hline
\end{tabular}

shown in Table 4, the strength of the rock mass with different angles is determined when $\sigma_{3}$ is between 0 and $0.2 \mathrm{MPa}$. The abovementioned method is used to determine the material parameters of each failure criterion. As shown in Figure 11, the results show that the empirical equations of NLEE and 


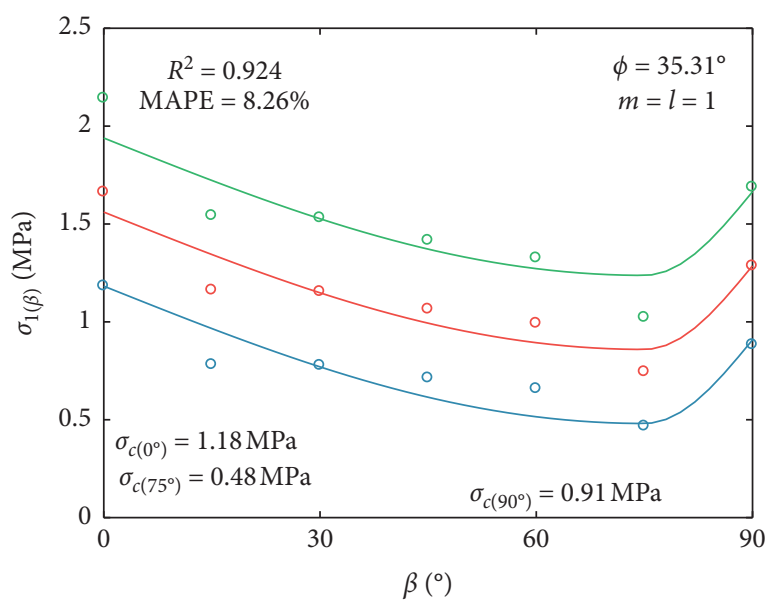

$$
\begin{aligned}
& \text { Predictive value: Distrate value: } \\
& -\sigma_{3}=0 \quad \circ \sigma_{3}=0 \\
& -\sigma_{3}=0.1 \mathrm{MPa} \quad \circ \sigma_{3}=0.1 \mathrm{MPa} \\
& -\sigma_{3}=0.2 \mathrm{MPa} \quad \circ \sigma_{3}=0.2 \mathrm{MPa}
\end{aligned}
$$

(a)

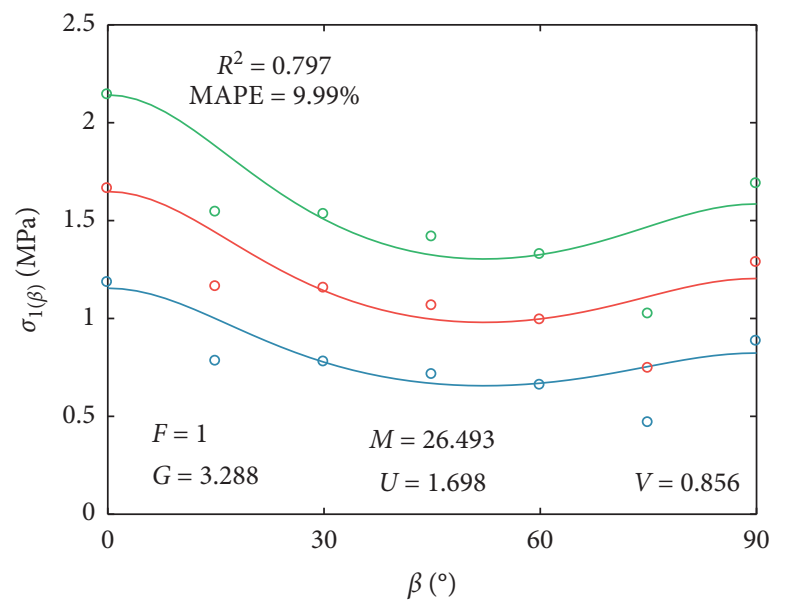

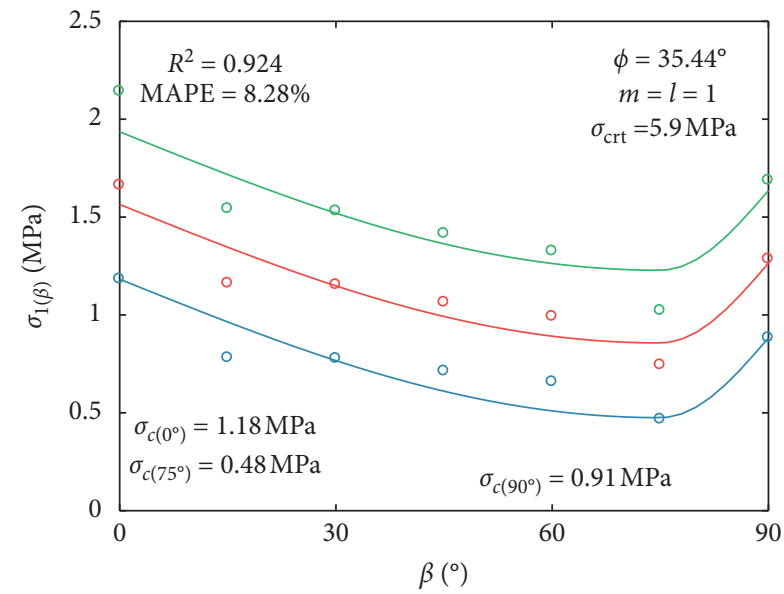

Predictive value:

$-\sigma_{3}=0$

$-\sigma_{3}=0.1 \mathrm{MPa}$

$-\sigma_{3}=0.2 \mathrm{MPa}$

Distrate value:

○ $\sigma_{3}=0$

○ $\sigma_{3}=0.1 \mathrm{MPa}$

$\sigma_{3}=0.2 \mathrm{MPa}$

(b)

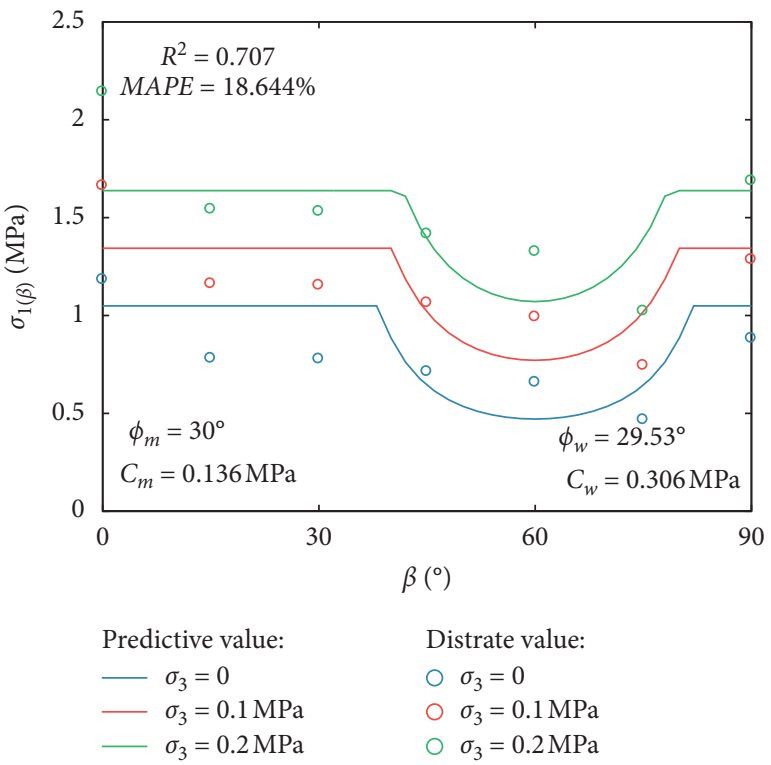

(d)

Figure 11: Predicted strength of the layered rock mass and the material coefficients of the four criteria. (a) LEE. (b) NLEE. (c) Pariseau $(n=1)$. (d) JPW.

LEE exhibit high accuracy. As a result, the elastic parameter of $E_{1}$ is $3.39 \mathrm{GPa}, E_{3}$ is $3 \mathrm{GPa}, G_{13}$ is $1.18 \mathrm{GPa}, u_{12}$ is 0.3 , and $u_{13}$ is 0.33 .

For analysis, the profile of the N26 exploration line is used. The boundary conditions that are applied are as follows: both the left and right boundaries are fixed in the horizontal direction and the bottom boundary of the domain is fixed in all directions. As shown in Figures 12(a) and 12(b), the gravity stress field of the slope and the angle of $\beta$ are calculated simultaneously based on the assumption of the plane strain of the finite-element method. The results demonstrate that the maximum principal stress of the slope surface is observed at a low-stress level. Moreover, the angle of $\beta$ is found to be close to the angle of $\theta\left(75^{\circ}\right)$ near the surface of some slope benches, indicating that shear-slip landslides can likely occur in these areas. Among them, the -18 to $-67 \mathrm{~m}$ bench is the area where shallow landslides actually occur.

As shown in Figure 13, four different failure criteria are used to examine the damage zone. Compared with the landslides with a thickness of $4 \mathrm{~m}$ of the slip body occurring on the actual $-67 \mathrm{~m}$ bench, the scale of the damage zone calculated by NLEE and LEE is the most consistent and that of the JPW and Pariseau $(n=1)$ is apparently smaller. The 


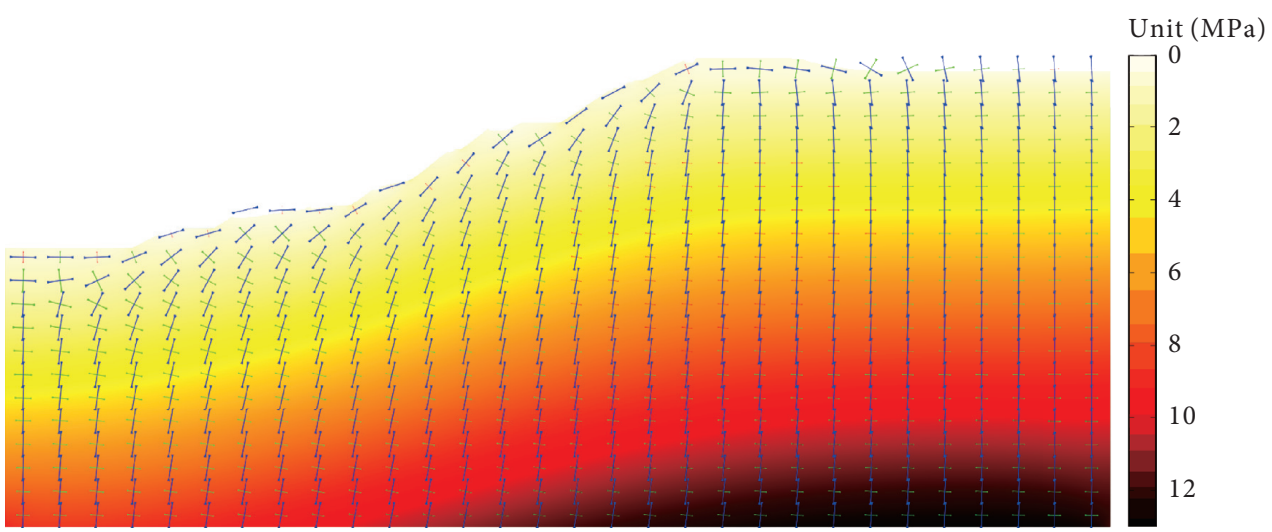

(a)

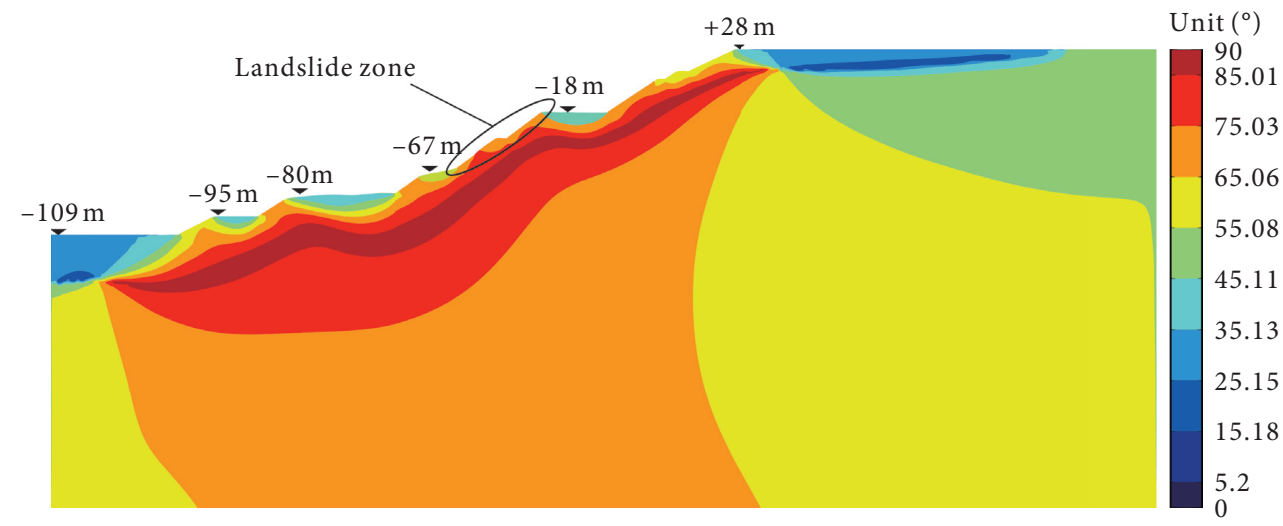

(b)

Figure 12: (a) The maximum principal stress distribution on the N26 profile. (b) The angle of $\beta$ distribution on the N26 profile.

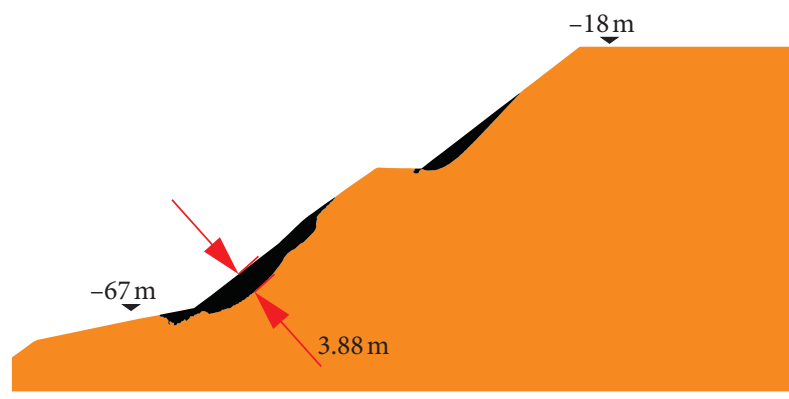

(a)

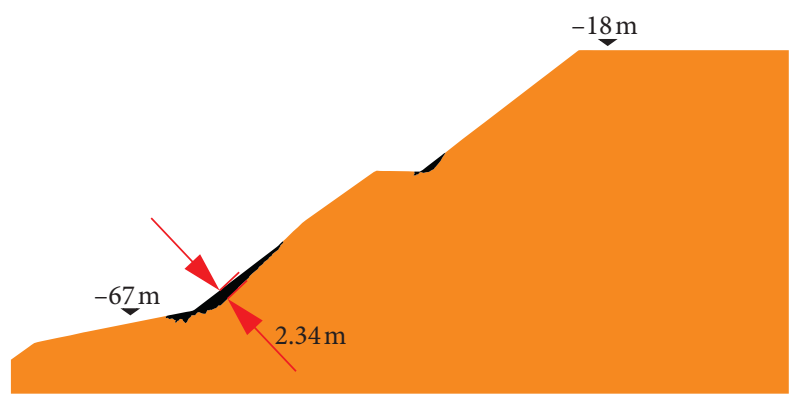

(c)

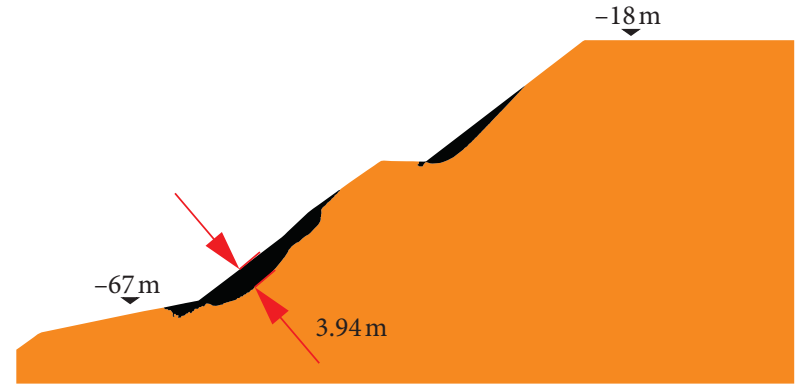

(b)

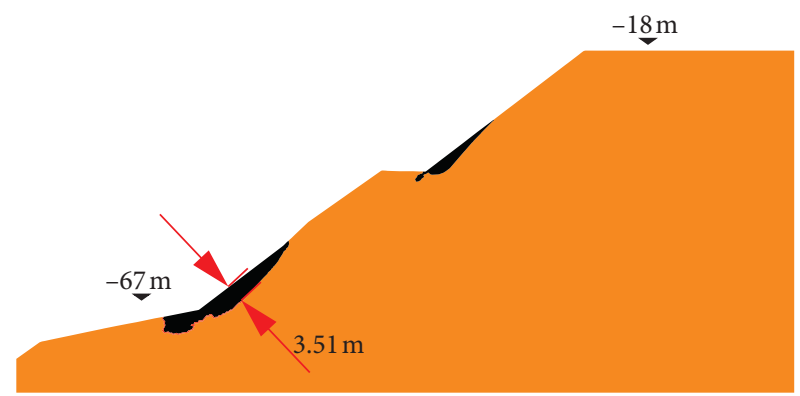

(d)

Figure 13: Damage zone of the slope based on four criteria. (a) LEE. (b) NLEE. (c) Pariseau ( $n=1)$. (d) JPW. 
results show that the prediction accuracy of different anisotropic criteria of the layered rock can also be applied in the layered slope engineering.

5.2. Case 2: Underground Powerhouse. The Jinping I Hydropower Station is located on the mainstream of the Yalong River in the Sichuan Province, China. As shown in Figure 14(a), the underground powerhouse is arranged at the right bank of the dam. The dimension of the main powerhouse is $204.52 \mathrm{~m} \times 25.90 \mathrm{~m} \times 68.83 \mathrm{~m}$, and the dimension of the main transformer chamber is $201.60 m \times 19.30 m \times 32.54 \mathrm{~m}$. The distance between the centers of the two caverns is estimated to be $67.35 \mathrm{~m}$. The surrounding rock in the underground powerhouse is primarily a bedded marble. The inclination of rock strata intersects with the small angle of the axis of the powerhouse. In addition, the apparent dip angle on the cross section of the primary factory building is found to be about $20^{\circ}$.

According to the result of the in situ stress test [65], the values of the maximum principal stress, the intermediate principal stress, and the minimum principal stress near the underground powerhouse are estimated to be 18.0-35.7 $\mathrm{MPa}, 10-24 \mathrm{MPa}$, and $3.65-14.45 \mathrm{MPa}$, respectively. For the numerical calculation, one set of typical stress data from the region of the powerhouse was selected. The plane stress system on the cross profile of the powerhouse $(y$ axis for vertical and $x$-axis for horizontal as shown in Figure 14(b)) was calculated by using the stress coordinate conversion method (Table 5).

A large number of studies have shown that the deformation and strength of rock mass show anisotropic characteristics due to the high in-situ stress and as an influence of structural planes [66]. However, due to the lack of strength test data of different dip angles of layered marble, in this study, the data are taken from the strength of the jointed marble of the Jinping II Hydropower Station [67] and the strength of the intact marble of the Jinping I Hydropower Station [65]. As shown in Table 6, for the latter, the value of $\beta$ is considered as $0^{\circ}$ or $90^{\circ}$. The elastic parameter of $E_{1}$ is $19.8 \mathrm{GPa}, E_{3}$ is $16.3 \mathrm{GPa}, G_{13}$ is $6.8 \mathrm{GPa}, u_{12}$ is 0.2 , and $u_{13}$ is 0.35 . Figure 15 shows the predicted strength of each failure criterion. Because of the lack of sufficient test data, NLEE demonstrates the highest prediction accuracy, whereas Pariseau $(n=1)$ demonstrates the lowest prediction accuracy.

The boundary condition is that the stress tensor in Table 4 is applied as initial stress in the whole region, and in the four sides, the model is subject to zero normal displacement. Based on the transversely isotropic elastic constitutive, the maximum principal stress distribution along the cross section of underground powerhouse and the angle of $\beta$ can be obtained (Figures 16(a) and 16(b)). Two compressive stress-concentrated regions are the upstream and the arch foot of the downstream of the main powerhouse and the main transformer room. In these regions, the angle of $\beta$ is estimated to be about $60^{\circ}$ and the strength is observed to be relatively low. Therefore, damage and failure can easily occur in these regions. Failure criteria are used to analyze the distribution of the damage zone around the underground powerhouse (see Figure 17). By using NLEE and LEE empirical equations, the results show the obvious damage zones in the downstream arch foot of the main powerhouse and the main transformer room. They are found to be in good agreement with the actual concrete lining damage and the bending deformation of reinforcing bars and shotcrete loss (see Figure 18(a)). The triangle damaged area appears in the roof arch, which is found to be consistent with the roof spalling in the layered roadway (see Figure 18(b)). However, the damage zone calculated by the Pariseau $(n=1)$ and the JPW criterion is estimated to be smaller, whereas the damage zone of the JPW criterion is found to be inconsistent with the actual situation. The results show that the prediction accuracy of different anisotropic criteria of the layered rock can also be applied in the layered underground powerhouse engineering.

\section{Discussion}

6.1. Influencing Factors to Predict the Accuracy of the Layered Rock Strength. Considering the quantitative evaluation of the prediction accuracy of the rock compressive strength based on different anisotropic failure criteria, four factors can be suggested that affect the prediction accuracy. According to the degree of influence, these factors are as follows: (1) the number of effective experimental data. The prediction accuracy of different failure criteria decreases with different degrees as the number of available data decreases. Taking into consideration the empirical equation and JPW, for example, the MAPE increases from less than $16 \%$ to less than $29 \%$ and the value of $R^{2}$ decreases from about 0.9 to about 0.5 . (2) The function type of the failure criterion. The function types of failure criterion are divided into continuous or piecewise functions and linear or nonlinear functions. For instance, the Pariseau criteria are continuous linear and nonlinear functions when $n=1$ and $n=2$, respectively. However, the latter is an exponential function, and its form does not conform to the strength law of layered rocks. Therefore, its fitting degree is extremely poor, and the $M A P E$ reaches $78 \%$, which is not suitable for layered rocks. However, empirical equations LEE and NLEE are considered as piecewise linear and nonlinear functions, respectively. The latter is modified on the basis of the former taking into consideration the nonlinear strength characteristics of rocks. The MAPE decreases by $2-4 \%$ and the value of $R^{2}$ increases. (3) The number of material parameters in the failure criterion. The strength law due to the complex failure mechanism of layered rocks requires to be characterized by multiple potential mechanical parameters. For instance, the prediction accuracy of the empirical formula, Pariseau's criterion $(n=1)$, and the JPW criterion is observed to be proportional to the number of material parameters with all available data. (4) The influence of the high confining pressure. The nonlinear strength characteristics of layered rocks lead to a decrease in the prediction accuracy of the strength criterion under the condition of high confining pressure. 


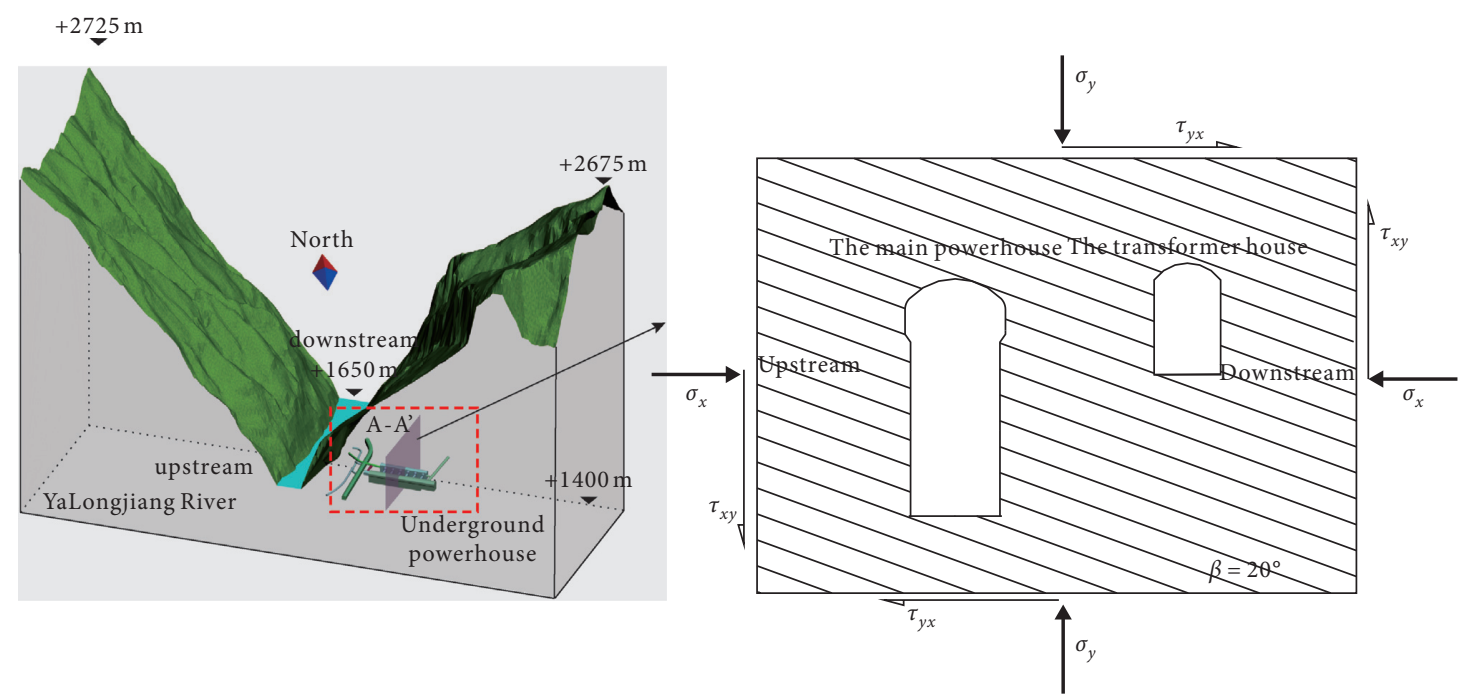

(a)

(b)

FIGURE 14: (a) 3D layout of Jinping I underground hydropower station caverns group. (b) The A- $\mathrm{A}^{\prime}$ profile of the main powerhouse and transformer house.

TABLE 5: In situ stress measuring points of underground powerhouse [65].

\begin{tabular}{lccccccc}
\hline ID & - & $\sigma_{1}$ & $\sigma_{2}$ & $\sigma_{3}$ & $\sigma_{x}$ & $\sigma_{y}$ & $\tau_{x y}$ \\
\hline \multirow{4}{*}{$\sigma 27-5$} & Value (MPa) & 23.21 & 17.67 & 10.59 & 14.98 & 19.72 \\
& Dip direction ( $\left.{ }^{\circ}\right)$ & 147.8 & 350.9 & 248.0 & 25 & 0.45 \\
& Dip angle ( $\left.{ }^{\circ}\right)$ & 40.5 & 47.1 & 11.7 & 0 & 25 \\
\hline
\end{tabular}

TABLE 6: Mechanical parameters of jointed marble and intact marble $[65,67]$.

\begin{tabular}{lccc}
\hline Type & $\beta\left(^{\circ}\right)$ & $\sigma_{3}$ & \\
\hline Jointed marble & 60.7 & 5 & $\sigma_{1}$ \\
& 40.6 & 5 & 47 \\
& 34 & 5 & 93.9 \\
& 42 & 10 & 95 \\
& 41 & 40 & 117.2 \\
Intact marble & 0 r 90 & 0 & 181.9 \\
& 0 or 90 & 20 & 75 \\
\hline
\end{tabular}

6.2. Suggestions of the Anisotropic Failure Criterion in Engineering. The strength test of the anisotropic rock takes more time and it is costly as compared to the isotropic rock. Moreover, it is difficult to perform a large number of triaxial tests on layered rocks of various lithologies in engineering. In order to determine the strength parameters for balancing economy and security, different numbers and types of experiments should be conducted according to the engineering characteristics of the layered rock mass, such as in situ stress level and engineering safety level. The empirical equation (LEE or NLEE) has a higher prediction accuracy because most rock mass engineering is performed under medium and low confining pressures, and therefore it is recommended to be used. Two specific suggestions are explained as follows.

(1) For shallow engineering, such as open-pit slope, the shear-slip failure along with the bedding is considered to be the most probable and the shallow failure is considered to be the main failure mode. The rock mass in the damage area mostly occurs at the lowstress level. Therefore, uniaxial and shear tests can be conducted for obtaining cohesion and friction angle parameters of the strength of layered rocks with three key angles of $\beta$ at $0^{\circ}, 90^{\circ}$, and $60^{\circ}-75^{\circ}$. The cost of the shear test is much lower as compared to that of the triaxial test. In order to calculate mechanical parameters of the rock mass at different angles on the basis of laboratory experiments, the Hoek-Brown criterion can be used for estimating mechanical parameters of the rock mass.

(2) For deep underground engineering, the failure criterion is generally considered in the superimposed environment of high tectonic stress and automatic stress. Generally, secondary stress forms a highstress concentration area around the rock mass after the unloading of underground engineering 

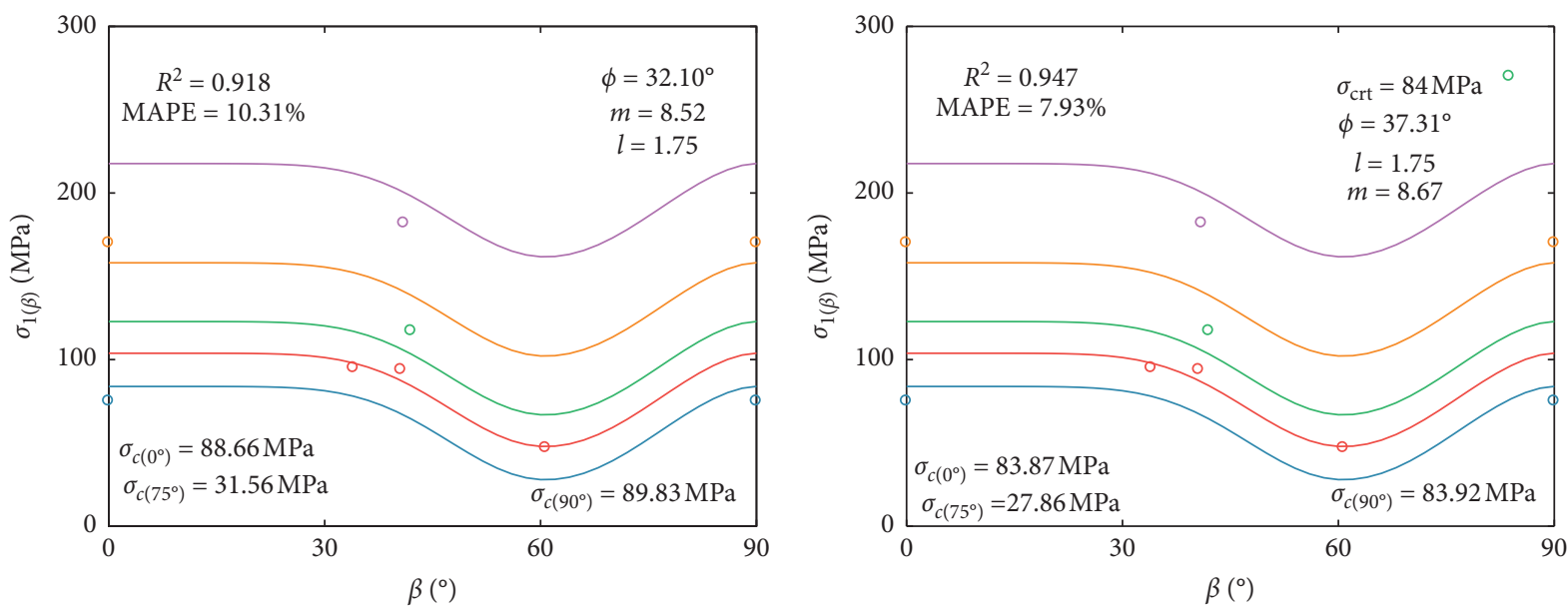

\begin{tabular}{|c|c|}
\hline $\begin{array}{c}\text { Predictive value: } \\
\qquad \sigma_{3}=0\end{array}$ & Distrate value: \\
\hline $\begin{array}{l}\sigma_{3}=0 \\
\sigma_{2}=5 \mathrm{MPa}\end{array}$ & $\sigma_{3}=0$ \\
\hline $\begin{array}{l}\sigma_{3}=5 \mathrm{NI} \\
\sigma_{3}=10 \mathrm{MPa}\end{array}$ & $\sigma_{3}=5 \mathrm{MIC}$ \\
\hline$\sigma_{3}=20 \mathrm{MPa}$ & $\sigma_{3}=20 \mathrm{MPa}$ \\
\hline$\sigma_{3}=40 \mathrm{MPa}$ & $\sigma_{3}=40 \mathrm{MPa}$ \\
\hline
\end{tabular}

(a)

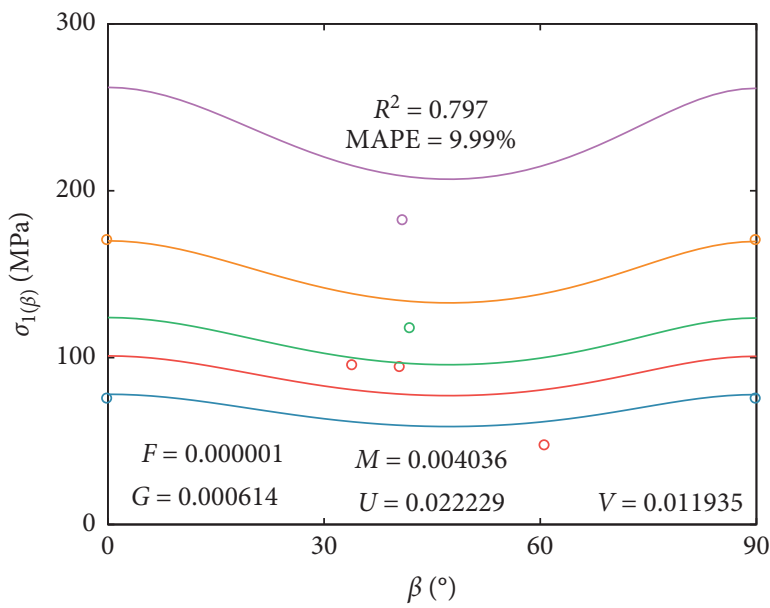

(c)
Distrate value:
○ $\sigma_{3}=0$
○ $\sigma_{3}=5 \mathrm{MPa}$
- $\sigma_{3}=10 \mathrm{MPa}$
- $\sigma_{3}=20 \mathrm{MPa}$
○ $\sigma_{3}=40 \mathrm{MPa}$

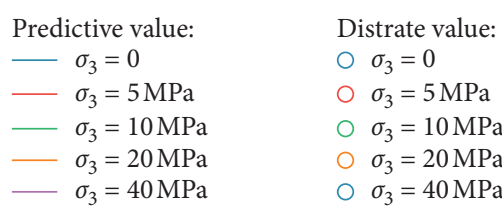

(b)

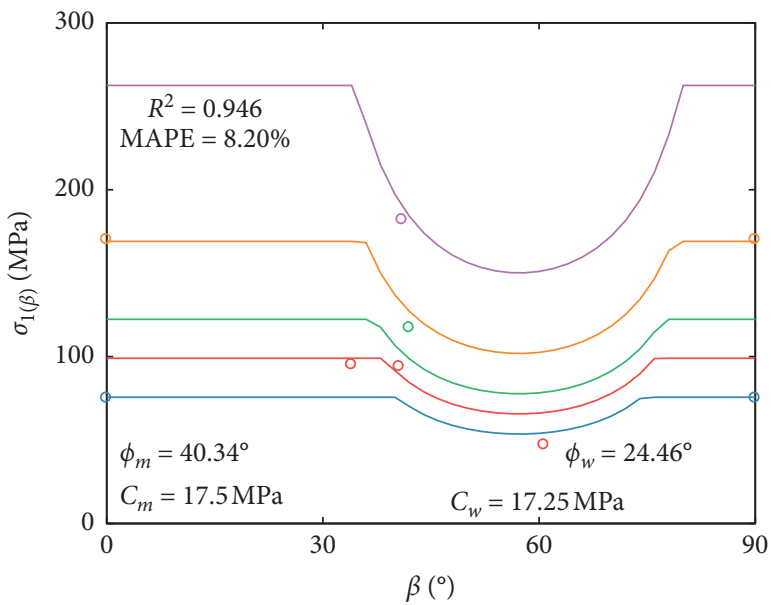

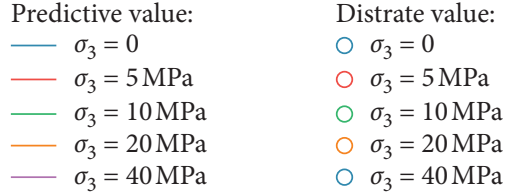

(d)

Figure 15: Predicted strength of the bedded marble and the material coefficients of the four criteria. (a) LEE. (b) NLEE. (c) Pariseau ( $n=1$ ). (d) JPW.

excavation. In order to ensure triaxiality under medium and low confining pressures, it is necessary to conduct uniaxial and conventional triaxial tests at different angles. The prediction accuracy of the axial strength requires the in situ stress measurement and the finite-element calculation for determining the confining pressure level.
6.3. Limitations and Future Work. In this study, there were some limitations. (1) Only the strength law under conventional triaxial conditions is discussed; however, the effect of intermediate principal stress is not considered. The intermediate principal $\sigma_{2}$ is considered based on the Pariseau's criterion. The influence of intermediate principal stress on the strength of the weak plane can be considered by the JPW. 


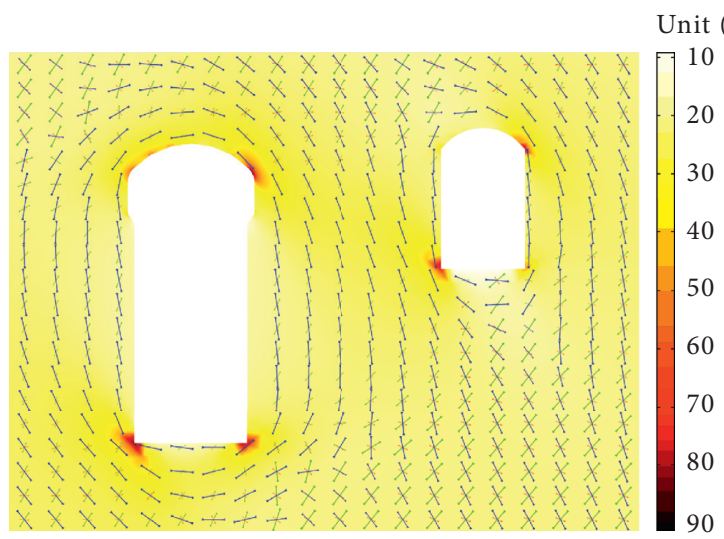

(a)

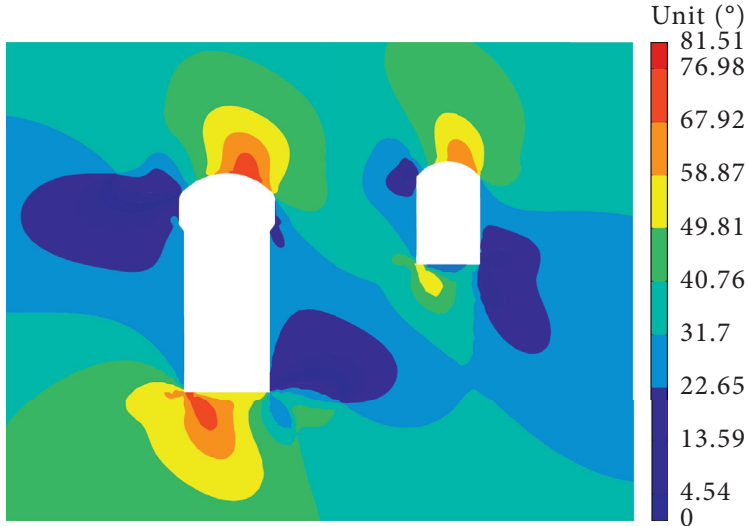

(b)

Figure 16: (a) The maximum principal stress distribution along the cross section of underground powerhouse. (b) The angle of $\beta$ distribution along the cross section of underground powerhouse.

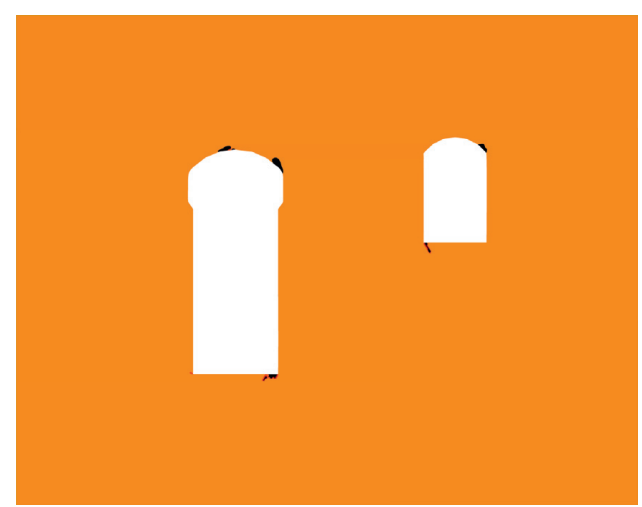

(a)

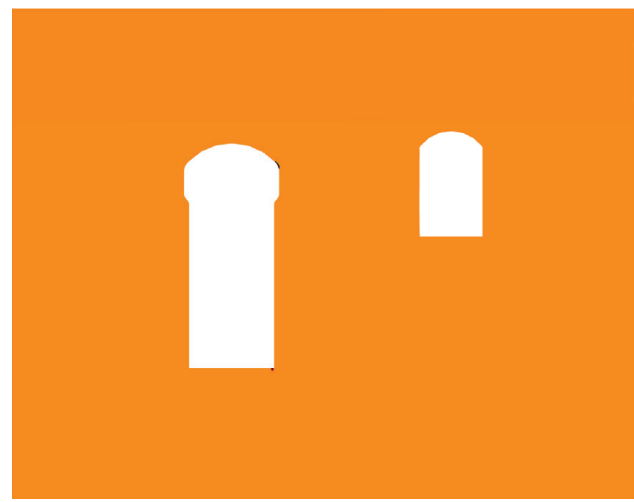

(c)

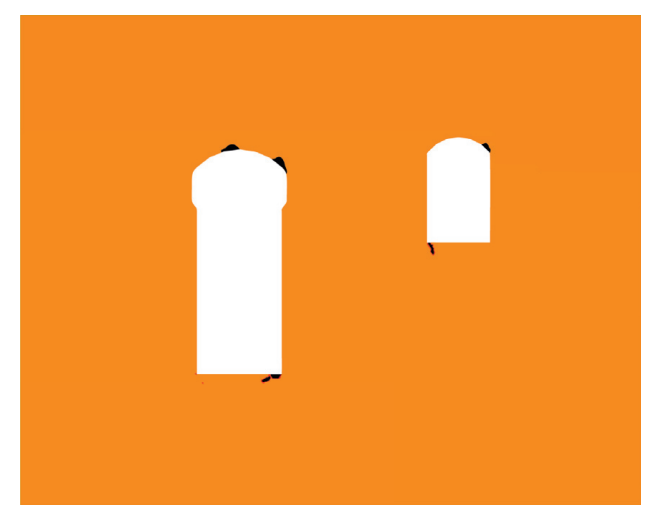

(b)

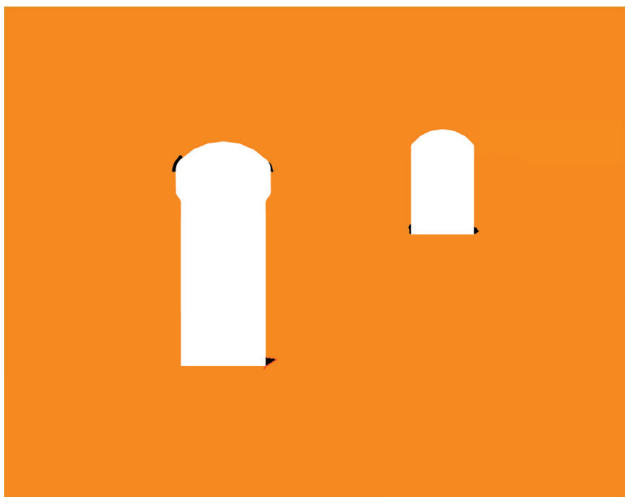

(d)

Figure 17: Damage zone of the underground powerhouse based on four criteria. (a) LEE. (b) NLEE. (c) Pariseau ( $n=1$ ). (d) JPW.

Both of them can be directly used in the strength prediction of true triaxial, whereas the empirical equation does not consider the influence of intermediate principal stress. Some true triaxial strength data are collected to predict Pariseau $(n=1)$ and JPW. The results show that both of them can reflect the variation in the true triaxial strength of the anisotropy rock [59]. (2) Only elastic stress condition is considered, while the damage development law based on anisotropic failure criteria is not considered further. Specifically, in deep engineering, rock mass damage deteriorates with high in situ stress to form a certain depth of a loosened circle. Therefore, the predicted accuracy of the anisotropic failure criteria will be evaluated based on true triaxial data in the future. Based on the anisotropic failure criteria, the numerical simulation of the elastic damage evolution of the rock mass will be further developed. 


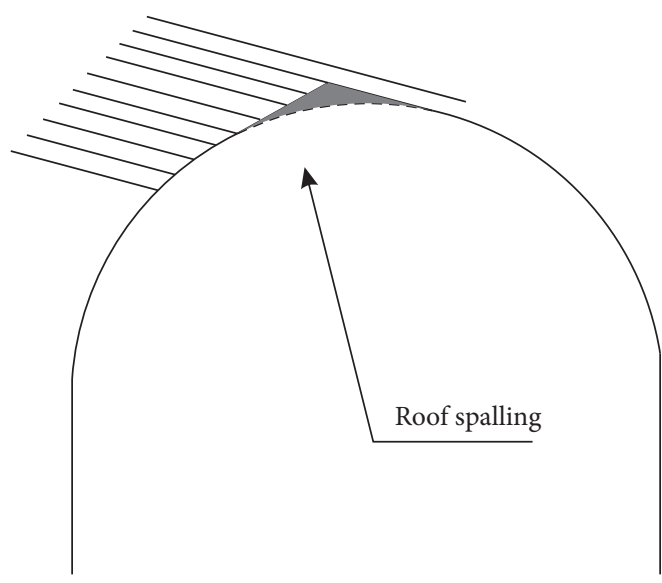

(a)

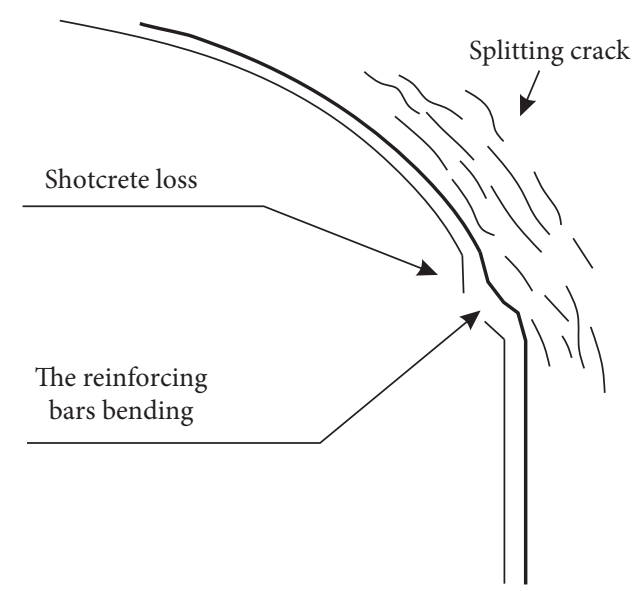

(b)

FIGURE 18: (a) The roof spalling around the roadway in 20 inclined layered roadway. (b) The bending deformation of reinforcing bars and shotcrete loss at the downstream sidewall of the crown in main powerhouse.

TABLE 7: The order of the predictive accuracy of failure criteria under different conditions.

\begin{tabular}{|c|c|c|c|}
\hline- & Low to high confining pressure & Medium to low confining pressure & High confining pressure \\
\hline All data available & & NLEE $>$ LEE $>$ Pariseau $>$ JPW & \\
\hline Part of data is available & NLEE $>(J P W \approx$ LEE $)>$ Pariseau & NLE $>$ LEE $>$ JPW $>$ Pariseau & JPW $>$ NLEE $>$ LEE $>$ Pariseau \\
\hline
\end{tabular}

\section{Conclusion}

(1) When $n=2$, the Pariseau's criterion (Hoffman criterion) is not considered suitable for the layered rock. When $n=1$, the fit of the Pariseau model will be worst among the four criteria without enough triaxial data, its $R^{2}$ value is estimated to be below 0.1 and MAPE fluctuates within $26 \%$.

(2) Under the condition of medium to low confining pressures, the empirical equations (LEE and NLEE) exhibit good predictive accuracy (MAPE <20\%). Under the condition of high confining pressure, the predictive accuracy of all criteria tends to decrease, and JPW is considered to be the best among all (MAPE $<40 \%$ ). Table 7 shows the list of the failure criteria in the order of predictive accuracy.

(3) Combining the verification of engineering application and the precision assessment of the four failure criteria, it is recommended to apply the empirical equations (LEE and NLEE) in most of the layered rock mass.

\section{Abbreviations}

B:

$\theta:$

$\sigma_{c}(\beta), \sigma_{c}(\theta):$
Angle between horizontal direction and the bedding plane direction The value of $\beta$ at minimum compressive strength, usually $45 \sim 60^{\circ}$ $\sigma_{\text {crt: }}$

$\phi:$

$\phi_{m} \phi_{w}:$

$c_{m}, c_{w}:$

$X_{t}, X_{c}, Y_{t}, Y_{c}:$

$S:$

$\sigma_{1}, \sigma_{3}:$

F, G, M, U, V, $n$ : Material constants of Pariseau's Criteria,

$m, l:$

$\sigma_{x x}, \sigma_{y y}, \sigma_{z z}, \sigma_{x y}$,

$\sigma_{z x}, \sigma_{z y}:$

MAPE:

$R^{2}$ :

$\sigma_{1 \operatorname{expr}}, \sigma_{\mathrm{cal}}:$
UCS of anisotropic rock with planes of anisotropy oriented at angle of $\beta$ and $\theta$ from horizontal direction

Critical confining pressure for the layered rock

The friction angle of the equivalent continuum of rock matrix and weakness Friction angle of rock matrix and weakness planes Cohesion of rock matrix and weakness planes

The uniaxial tension and compression strengths in the directions normal and parallel to the bedding planes The pure shear strength of the rock mass along weak planes Major and minor principal stresses at failure where $n \geq 1$ Material constants of linear and nonlinear empirical equation The stress component under material direction Mean absolute percentage error The goodness of fit Experimental and predicted values of the major principal stresses at failure 

$\sigma_{1 a v}:$
Average of the experimental $\sigma_{1}$ values for a triaxial dataset
$N$ :
The numbers of the dataset.

\section{Data Availability}

The complete database and the complete programs are available on GitHub. (https:/github.com/Dwxasd/Anisotropic-FailureCriteria)

\section{Conflicts of Interest}

The authors declare that they have no conflicts of interest.

\section{Acknowledgments}

This work was financially supported by the Natural Science Foundation of China (Grant nos. 51574059 and U1710253) and the China National Key R\&D Program (Grant nos. 2016YFC0801602 and 2017YFC1503101).

\section{References}

[1] M. H. B. Nasseri, K. S. Rao, and T. Ramamurthy, "Anisotropic strength and deformational behavior of himalayan schists," International Journal of Rock Mechanics and Mining Sciences, vol. 40, no. 1, pp. 3-23, 2003.

[2] H. Niandou, J. F. Shao, J. P. Henry, and D. Fourmaintraux, "Laboratory investigation of the mechanical behaviour of tournemire shale," International Journal of Rock Mechanics and Mining Sciences, vol. 34, no. 1, pp. 3-16, 1997.

[3] Y. M. Tien and M. C. Kuo, "A failure criterion for transversely isotropic rocks," International Journal of Rock Mechanics and Mining Sciences, vol. 38, no. 3, pp. 399-412, 2001.

[4] Y. Zhao, C. Wang, Y. Zhang, and Q. Liu, "Experimental study of adsorption effects on shale permeability," Natural Resources Research, vol. 28, no. 4, pp. 1575-1586, 2019.

[5] M. Singh, N. K. Samadhiya, A. Kumar, V. Kumar, and B. Singh, "A nonlinear criterion for triaxial strength of inherently anisotropic rocks," Rock Mechanics and Rock Engineering, vol. 48, no. 4, pp. 1387-1405, 2015.

[6] H. Lee, S. H. Ong, M. Azeemuddin, and H. Goodman, "A wellbore stability model for formations with anisotropic rock strengths," Journal of Petroleum Science and Engineering, vol. 96-97, pp. 109-119, 2012.

[7] B. Amadei, "Importance of anisotropy when estimating and measuring in situ stresses in rock," International Journal of Rock Mechanics and Mining Sciences \& Geomechanics Abstracts, vol. 33, no. 3, pp. 293-325, 1996.

[8] J. J. C. Shear, "Failure of anistropic rocks," Geological Magazine, vol. 97, no. 1, pp. 65-72, 1960.

[9] J. B. Walsh and W. F. Brace, "A fracture criterion for brittle anisotropic rock," Journal of Geophysical Research, vol. 69, no. 16, pp. 3449-3456, 1964.

[10] R. Mclamore and K. E. Gray, "The mechanical behavior of anisotropic sedimentary rocks," Journal of Engineering for Industry, vol. 89, no. 1, p. 62, 1967.

[11] F. A. Donath, "Experimental study of shear failure in anisotropic rocks," Geological Society of America Bulletin, vol. 72, no. 6, pp. 985-989, 1961.

[12] M. E. Chenevert and C. Gatlin, "Mechanical anisotropies of laminated sedimentary rocks," Society of Petroleum Engineers Journal, vol. 5, no. 1, pp. 67-77, 1965.
[13] F. D. Itasca, Fast Lagrangian Analysis of Continua in 3-dimensions, Version 3.0, Manual, Itasca Consulting Group, Inc., Itasca, Minnesota, 1997.

[14] G. Duveau, J. F. Shao, and J. P. Henry, "Assessment of some failure criteria for strongly anisotropic geomaterials," $\mathrm{Me}$ chanics of Cohesive-Frictional Materials, vol. 3, no. 1, pp. 1-26, 1998.

[15] O. Hoffman, "The brittle strength of orthotropic materials," Journal of Composite Materials, vol. 1, no. 2, pp. 200-206, 1967.

[16] W. G. Pariseau, "Plasticity theory for anisotropic rocks and soil," in proceedings of the 10th US Symposium on Rock Mechanics, Golden, Colorado, October 1968.

[17] S. W. Tsai and E. M. Wu, "A general theory of strength for anisotropic materials," Journal of Composite Materials, vol. 5, no. 1, pp. 58-80, 1971.

[18] H. Saroglou and G. Tsiambaos, "A modified Hoek-Brown failure criterion for anisotropic intact rock," International Journal of Rock Mechanics and Mining Sciences, vol. 45, no. 2, pp. 223-234, 2008.

[19] L. Zhang and H. Zhu, "Three-dimensional Hoek-Brown strength criterion for rocks," Journal of Geotechnical and Geoenvironmental Engineering, vol. 133, no. 9, pp. 1128-1135, 2007.

[20] Y.-K. Lee, S. Pietruszczak, and B.-H. Choi, "Failure criteria for rocks based on smooth approximations to mohr-coulomb and hoek-brown failure functions," International Journal of Rock Mechanics and Mining Sciences, vol. 56, pp. 146-160, 2012.

[21] Y. Xu, M. Ying-Feng, L. Gao et al., "An empirical equation to estimate uniaxial compressive strength for anisotropic rocks," Rock Soil Mechanics, vol. 38, no. 9, 2017.

[22] T. Ramamurthy, G. Venkatappa Rao, and J. Singh, "A strength criterion for anisotropic rocks," in Proceedings of the Australia-New Zealand Conference on Geomechanics, 5th, 1988, Sydney, Australia, March 1988.

[23] R. V. Mises, "Mechanik der plastischen formänderung von kristallen," ZAMM-Zeitschrift für Angewandte Mathematik und Mechanik, vol. 8, no. 3, pp. 161-185, 1928.

[24] A. Casagrande and N. Carrillo, "Discussion on study of failure envelope soils," Journal of the Soil Mechanics, ASCE, vol. 89, p. 243, 1944.

[25] J. C. Jaeger, Elasticity, Fracture and Flow: with Engineering and Geological applications, Springer Science \& Business Media, Berlin, Germany, 2012.

[26] R. Hill, "A theory of the yielding and plastic flow of anisotropic metals," Proceedings of the Royal Society of London. Series A. Mathematical and Physical Sciences, vol. 193, no. 1033, pp. 281-297, 1948.

[27] W. Olszak and W. Urbanowski, "The plastic potential and the generalized distortion energy in the theory of non-homogeneous anisotropic elastic-plastic bodies," Archives of $\mathrm{Me}$ chanics, vol. 8, no. 4, pp. 671-694, 1956.

[28] R. Mclamore and K. Gray, "A strength criterion for anisotropic rocks based upon experimental observations," Annual Meeting of the American Institute of Mining, Metallurgical, and Petroleum Engineers, vol. 21, 1967.

[29] E. Hoek, "Fracture of anisotropic rock," Journal of the South African Institute of Mining Metallurgy, vol. 64, no. 10, pp. 501-523, 1964.

[30] I. Goldenblat, Some Problems of the Mechanics of Continuous media, Noordhoff, Groningen, Netherlands, 1962.

[31] E. Hoek and E. T. Brown, "Empirical strength criterion for rock masses," Journal of Geotechnical Geoenvironmental Engineering, vol. 7, p. 106, 1980. 
[32] I. Goldenblat and V. Kopnov, "A generalized theory of plastic flow of anisotropic metals," Stroitelnaya Mekhanika, vol. 307, p. 319, 1966.

[33] H. A Ashour, "A compressive strength criterion for anisotropic rock materials," Canadian Geotechnical Journal, vol. 25, no. 2, pp. 233-237, 1988.

[34] S. A. F. Murrell, "The effect of triaxial stress systems on the strength of rocks at atmospheric temperatures," Geophysical Journal International, vol. 10, no. 3, pp. 231-281, 1965.

[35] D.-Q. Zhou, X.-S. Liu, and L. Qi, "Strength criteria for anisotropic rocks and experimental studies," 1992.

[36] K. Barron, "Brittle fracture initiation in and ultimate failure of rocks," International Journal of Rock Mechanics and Mining Sciences \& Geomechanics Abstracts, vol. 8, no. 6, pp. 553-563, 1971.

[37] B. Single, R. K. Goel, V. K. Mehrotra, S. K. Garg, and M. R. Allu, "Effect of intermediate principal stress on strength of anisotropic rock mass," Tunnelling and Underground Space Technology, vol. 13, no. 1, pp. 71-79, 1998.

[38] G. Archambault and B. Ladanyi, "Failure of jointed rock masses by kink zone instability: an important and dangerous mode of rupture," ISRM International Symposium-EUROCK, vol. 93, 1993.

[39] Z. T. Bieniawski, "Estimating the strength of rock materials," Journal of the Southern African Institute of Mining Metallurgy, vol. 74, no. 8, pp. 312-320, 1974.

[40] R. Nova and G. Sacchi, A generalized failure condition for orthotropic solids, Mechanical Behavior of Anisotropic Solids/ Comportment Méchanique des Solides Anisotropes, Springer, Berlin, Germany, 1982.

[41] K. Colak and T. Unlu, "Effect of transverse anisotropy on the Hoek-Brown strength parameter 'mi' for intact rocks," International Journal of Rock Mechanics and Mining Sciences, vol. 41, no. 6, pp. 1045-1052, 2004.

[42] M. B. Smith and J. B. Cheatham, "An anisotropic compacting yield condition applied to porous limestone," International Journal of Rock Mechanics and Mining Sciences \& Geomechanics Abstracts, vol. 17, no. 3, pp. 159-165, 1980.

[43] Y. F. Dafalias, "Anisotropic hardening of initially orthotropic materials," ZAMM-Zeitschrift für Angewandte Mathematik und Mechanik, vol. 59, no. 9, pp. 437-446, 1979.

[44] Y. Dafalias, "An anisotropic critical state clay plasticity model," in proceedings of the Second International Conference on Constitutive Laws for Engineering Materials-Theory and Applications, New York, N.Y, USA, December 1987.

[45] R. Yoshinaka and T. Yamabe, "A strength criterion of rocks and rock masses," ISRM International Symposium, Turin, Italy, 1981.

[46] D. Allirot, J. P. Boehler, and A. Sawczuk, "Irreversible deformations of an anisotropic rock under hydrostatic pressure," International Journal of Rock Mechanics and Mining Sciences \& Geomechanics Abstracts, vol. 14, no. 2, pp. 77-83, 1977.

[47] J. Pei, Strength of Transversely Isotropic rocks, Massachusetts Institute of Technology, Cambridge, MA, USA, 2008.

[48] R. Nova, "The failure of transversely isotropic rocks in triaxial compression," International Journal of Rock Mechanics and Mining Sciences \& Geomechanics Abstracts, vol. 17, no. 6, pp. 325-332, 1980.

[49] G.-Q. Zhang, "Rock failure with weak planes by self-locking concept," International Journal of Rock Mechanics and Mining Sciences, vol. 46, no. 6, pp. 974-982, 2009.
[50] J. Boehler and J. Raclin, "Anisotropic hardening of prestrained rolled sheet-steel," Current Advances in Mechanical Design Production, vol. 53, pp. 483-492, 1982.

[51] Y.-K. Lee and S. Pietruszczak, "A new numerical procedure for elasto-plastic analysis of a circular opening excavated in a strain-softening rock mass," Tunnelling and Underground Space Technology, vol. 23, no. 5, pp. 588-599, 2008.

[52] J. Raclin, "Contribution théorique et expérimentale à l'étude de l'écrouissage et de la rupture des solides anisotropes," thesis, University of Grenoble, Grenoble, France, 1984.

[53] D. G. Karr, F. P. Law, M. H. Fatt, and G. F. N. Cox, "Asymptotic and quadratic failure criteria for anisotropic materials," International Journal of Plasticity, vol. 5, no. 4, pp. 303-336, 1989.

[54] O. Cazacu and N. D. Cristescu, "A paraboloid failure surface for transversely isotropic materials," Mechanics of Materials, vol. 31, no. 6, pp. 381-393, 1999.

[55] M. Kusabuka, H. Takeda, H. Kojo, and T. Tonegawa, "Anisotropic yield function for rocks and evaluation of material parameters," Doboku Gakkai Ronbunshu, vol. 1999, no. 631, pp. 205-220, 1999.

[56] S. Pietruszczak and Z. Mroz, "On failure criteria for anisotropic cohesive-frictional materials," International Journal for Numerical and Analytical Methods in Geomechanics, vol. 25, no. 5, pp. 509-524, 2001.

[57] C. Xin and Y. Qiang, "Anisotropic yield criterion based on microplane effective stress vector," Acta Mechanica SinicaChinese Edition, vol. 38, no. 5, p. 692, 2006.

[58] Z. Mróz and J. Maciejewski, Critical Plane Approach to Analysis of Failure Criteria for Anisotropic geomaterials,Bifurcations, Instabilities and Degradations in Geomaterials, Springer, Berlin, Germany, 2011.

[59] J. Ambrose, Failure of Anisotropic Shales under Triaxial Stress Conditions, Imperial College London, London, England, 2014.

[60] B. Amadei, "Rock anisotropy and the theory of stress measurements," 1983.

[61] P. Wang, T. Yang, and J. Zhou, "Slope failure analysis considering anisotropic characteristics of foliated rock masses," Arabian Journal of Geosciences, vol. 11, no. 9, p. 222, 2018.

[62] D. Huafeng, W. Wei, L. Jianlin et al., "Experimental study on anisotropic characteristics of bedded sandstone," Chinese Journal of Rock Mechanics and Engineering, vol. 37, no. 1, pp. 112-120, 2018.

[63] T. Chen, X. Feng, X. Zhang et al., "Experimental study on mechanical and anisotropic properties of black shale," Chinese Journal of Rock Mechanics and Engineering, vol. 33, no. 9, pp. 1772-1779, 2014.

[64] W. Mu, L. Li, T. Yang et al., "Numerical investigation on a grouting mechanism with slurry-rock coupling and shear displacement in a single rough fracture," Bulletin of Engineering Geology the Environment, vol. 4, pp. 1-19, 2019.

[65] L. U. Bo, J. Wang, X. Ding et al., "Study of deformation and cracking mechanism of surrounding rock of jinping I underground powerhouse," Chinese Journal of Rock Mechanics and Engineering, vol. 29, no. 12, pp. 2429-2441, 2010.

[66] S. Huang, J. Wang, X. Ding et al., "Stability and control for underground caverns of jinping I hydropower station based on unloading evolution of layered rockmass," Chinese Journal of Rock Mechanics and Engineering, vol. 30, no. 11, pp. 2203-2216, 2011.

[67] L. I. Hongzhe, C. Xia, X. Wang et al., "Experimental study on deformation and strength properties of jointed marble specimens," Chinese Journal of Rock Mechanics and Engineering, vol. 27, no. 10, pp. 2118-2123, 2008. 